\title{
Computation of the quantum effects due to a four-dimensional pseudoparticle*
}

\author{
G. 't Hooft ${ }^{\dagger}$ \\ Physics Laboratories, Harvard University, Cambridge, Massachusetts 02138 \\ (Received 28 June 1976)
}

\begin{abstract}
A detailed quantitative calculation is carried out of the tunneling process described by the Belavin-PolyakovSchwarz-Tyupkin field configuration. A certain chiral symmetry is violated as a consequence of the AdlerBell-Jackiw anomaly. The collective motions of the pseudoparticle and all contributions from single loops of scalar, spinor, and vector fields are taken into account. The result is an effective interaction Lagrangian for the spinors.
\end{abstract}

\section{INTRODUCTION}

When one attempts to construct a realistic gauge theory for the observed weak, electromagnetic, and strong interactions, one is often confronted with the difficulty that most simple models have too much symmetry. In Nature, many symmetries are slightly broken, which leads to, for instance, the lepton masses, the quark masses, and $C P$ violation. These symmetry violations, either explicit or spontaneous, have to be introduced artificially in the existing models.

There is one occasion where explicit symmetry violation is a necessary consequence of the laws of relativistic quantum theory: the Adler-Bell-Jackiw anomaly. The theory we consider is an $\mathrm{SU}(2)$ gauge theory with an arbitrary set of scalar fields'and a number, $N^{f}$, of massless fermions. The apparent chiral symmetry of the form $\mathrm{U}\left(N^{f}\right)$

$\times \mathrm{U}\left(N^{f}\right)$ is actually broken down to $\mathrm{SU}\left(N^{f}\right) \times \mathrm{SU}\left(N^{f}\right)$

$\times U(1)$. This paper is devoted to a detailed computation of this effect.

The most essential ingredient in our theory is the localized classical solution of the field equations in Euclidean space-time, of the type found by Belavin et al. ${ }^{1}$

Although the main objective of this paper is the computation of the resulting effective symmetrybreaking Lagrangian in a weak-interaction theory, we present the calculations in such a way that they can also be used for possible color gauge theories of stong interactions based on the same classical field configurations. For such theories our intermediate expressions (12.5) and (12.8) will be applicable. Our final results are (15.1) together with the convergence factor (15.8).

Our general philosophy has been sketched in

Ref. 2. We are dealing with amplitudes that depend on the coupling constant $g$ in the following way:

$$
g^{-C} \exp \left[-\frac{8 \pi^{2}}{g^{2}}\left(1+a_{1} g^{2}+\cdots\right)\right] \text {. }
$$

The coefficient $a_{1}$ involves one-loop quantum cor- rections, and it determines the scale of the amplitude. Clearly, then, to understand the main features of such an amplitude, complete understanding of all one-loop quantum effects is desired. For instance, if one changes from one renormalization subtraction procedure to another, so that $g^{2} \rightarrow g^{2}+O\left(g^{4}\right)$, then this leads to a change in (1.1) by an overall multiplicative constant. Thus, the renormalization subtraction point $\mu$ may enter as a dimensional parameter in front of our expressions. This is just one of the reasons to suggest that our results will also have interesting applications in strong-interaction color gauge theories.

The underlying classical solutions only exist in Euclidean space, but they give rise to a particular symmetry-breaking amplitude that can easily be continued analytically to Minkowski space. We interpret this amplitude as the result of a certain tunneling effect from one vacuum to a gauge-rotated vacuum. We recall that, indeed, tunneling through a barrier can sometimes be described by means of a classical solution of the equation of motion in the imaginary time direction. ${ }^{2,3}$

We compute in Euclidean space the vacuum-tovacuum amplitude in the presence of external sources, thus obtaining full Green's functions. Of course, we must limit ourselves to gaugeinvariant sources only, but that will be no problem. It turns out to be trivial to amputate the obtained Green's function and get the effective vertex.

The various calculational steps are the following. We first give in Sec. II the functional integral expression for the amplitude, first in a conventional Feynman gauge: $C_{1}=\partial_{\mu} A_{\mu}$. Later, we go over to the so-called background gauge: $C_{4}=D_{\mu} A_{\mu}^{\mathrm{qu}}$. This is actually only correct up to an overall factor, as will be explained in Sec. XI. It is just for pedagogical reasons that we ignore this complication for a moment. It is in this gauge that the quantum excitations take a simple form: "Spinorbit" couplings commute with the operator $L^{2}$ 
$=-\frac{1}{8} L_{\mu \nu} L_{\mu \nu}$, where

$$
L_{\mu \nu}=x_{\mu} \frac{\partial}{\partial x_{\nu}}-x_{\nu} \frac{\partial}{\partial x_{\mu}},
$$

so that we can look at eigenstates of $L^{2}$. (In other gauges only total angular momentum $\overrightarrow{\mathrm{J}}=\overrightarrow{\mathrm{L}}+$ spin + isospin is conserved, not $L^{2}$.)

In Sec. III we consider the quantum fluctuations described by an eigenvalue equation,

$$
\mathfrak{M} \psi=E \psi,
$$

in order to compute det9lx. Now this looks like an ordinary scattering problem (in $4+1$ dimensions), and, indeed, we show that the product of all nonzero eigenvalues $E$, in some large box, can be expressed in terms of the phase shift $\eta(k)$ as a function of the wave number $k$.

In Sec. IV we show that Eq. (1.3) is essentially the same for scalars, spinors, and vectors, from which we derive the important result that the product of all nonzero eigenvalues is the same for scalar fields as for each component of the spinor and vector fields. So, we turn to the (much easier) scalar case first.

But even for scalars Eq. (1.3) has no simple solutions in terms of well-known elementary functions. We decide not to compute det?T by solving (1.2), but we compute instead

$$
\operatorname{det}\left[\left(1+x^{2}\right) \mathfrak{M}\left(1+x^{2}\right)\right] \text {. }
$$

The factors $1+x^{2}$ drop out if we divide by the same determinant coming from the vacuum (i.e., the case $A^{\mathrm{c} 1}=0$ ). The equation

$$
\mathfrak{M} \psi=\frac{\lambda}{\left(1+x^{2}\right)^{2}} \psi
$$

is a simple hypergeometric equation that can be solved under the given boundary condition (Sec. V).

Now we must find the product of all eigenvalues $\lambda$, but that diverges badly even if we divide by the values they take in the vacuum. We must find a gauge-invariant regulator, and the regulator determinant must be calculable. Dimensional regularization is not applicable here, but we can use background Pauli-Villars regulators. They give messy equations unless they have a spacetime-dependent mass:

$$
\frac{M_{i}^{2}}{\left(1+x^{2}\right)^{2}} \text {. }
$$

The rules are formulated in Sec. V.

In Sec. VI we compute the product of the eigenvalues using this regulator. In Sec. VII we make the transition to regulators with fixed mass by observing that a change toward fixed regulator mass must correspond to a local, space-time-dependent counterterm in the Lagrangian. The effect of this counterterm is computed.

Using the result of Sec. IV we now find also the contributions of all nonvanishing eigenvalues for the vectors and spinors. But there are also vanishing eigenvalues. They are listed in Sec. VIII. For the vector fields, we have eight zero eigenvectors in addition to the ones computed via the theorem of Sec. IV. They are to be interpreted as translations (Sec. IX), dilatation (Sec. X), and isospin rotations (Sec. XI). The last need special care and can only be interpreted correctly when different gauge choices are compared. This leads to the factor mentioned in the beginning.

In Sec. XII we combine the results so far obtained and add the fermions. This intermediate result may be useful to strong-interaction theories. In Sec. XIII we reexpress the result in terms of the dimensionally renormalized coupling constant $g^{D}$, as opposed to the previous coupling constant which was renormalized in a Pauli-Villars manner. In Sec. XIV the external sources for the fermions are considered and the amputation operation for the Green's function is performed. We obtain the desired effective Lagrangian, but there is still one divergence. So far, we only had massless particles, and as a consequence of that there is still a scale parameter $\rho$ over which we must integrate. Asymptotic freedom gives a natural cutoff for this integral in the ultraviolet direction, but there is still an infrared divergence. In weak-interaction theories the Higgs field is expected to provide for the infrared cutoff. Section XV shows how to compute this cutoff.

The Appendix lists the properties of the symbols $\eta, \bar{\eta}$ which are used many times throughout these calculations.

\section{FORMULATION OF THE PROBLEM}

Let a field theory in four space-time dimensions be given by the Lagrangian

$$
\mathscr{L}=-\frac{1}{4} G_{\mu \nu}^{a} G_{\mu \nu}^{a}-D_{\mu} \Phi^{*} D_{\mu} \Phi-\bar{\psi} \gamma_{\mu} D_{\mu} \psi+\bar{\psi}_{s} \mathcal{\partial}_{s t} \psi_{t},
$$

where the gauge group is $\mathrm{SU}(2)$ :

$$
G_{\mu \nu}^{a}=\partial_{\mu} A_{\nu}^{a}-\partial_{\nu} A_{\mu}^{a}+g \epsilon_{a b c} A_{\mu}^{b} A_{\nu}^{c} .
$$

The SU(2) indices will be called isospin indices. The scalars $\Phi$, taken to be complex, may contain several multiplets of arbitrary isospin:

$$
\begin{aligned}
& D_{\mu} \Phi=\partial_{\mu} \Phi-i g T^{a} A_{\mu}^{a} \Phi, \\
& {\left[T^{a}, T^{b}\right]=i \epsilon_{a b c} T^{c} .}
\end{aligned}
$$

The spinors $\psi$ are taken to be isospin- $\frac{1}{2}$ doublets. 
The total number of doublets is $N^{f}$. Mass terms and interaction terms between scalars and spinors are irrelevant for the time being.

We inserted a source term in a gauge-invariant way, with respect to which we will expand, in order to obtain Green's functions. The indices $s, t=1, \ldots, N^{f}$, called flavor indices, label the different isospin multiplets. Isospin and Dirac indices have been suppressed. $\mathcal{J}_{s t}$ must be diagonal in the isospin indices but may contain Dirac $\gamma$ matrices.

The system (2.1) seems to have a chiral $\mathrm{U}\left(N^{f}\right)$ $\times \mathrm{U}\left(N^{f}\right)$ global symmetry, but actually has an AdlerBell-Jackiw anomaly ${ }^{4}$ associated with the chiral U(1) current, breaking the symmetry down to $\mathrm{SU}\left(N^{f}\right)$ $\times \mathrm{SU}\left(N^{f}\right) \times \mathrm{U}(1)$. The aim of this paper is to find that part of the amplitude that violates the chiral $\mathrm{U}(1)$ conservation.

The functional integral expression for the amplitude is

$$
\begin{aligned}
W={ }_{\text {out }} & \langle 0 \mid 0\rangle_{\text {in }} \\
= & \int \mathbb{D} A \mathfrak{D} \psi \mathfrak{D} \Phi \mathfrak{D} \phi \\
& \quad \times \exp \left\{\int\left[\mathcal{L}-\frac{1}{2} C_{1}{ }^{2}(A)+\mathcal{L}_{1}^{\text {ghost }}(\phi)\right] d^{4} x\right\},
\end{aligned}
$$

to be expanded with respect to $\mathcal{J}$. Here $C_{1}(A)$ is a gauge-fixing term, and $\mathcal{L}_{1}^{\text {ghost }}$ are the corresponding ghost terms.

As is argued in Ref. 2, the U(1)-breaking part of this amplitude comes from that region of superspace where the $A$ field approaches the solutions described in Ref. 1:

$$
A_{\mu}^{a}(x)^{\mathrm{c} 1}=\frac{2}{g} \frac{\eta_{a \mu \nu}(x-z)^{\nu}}{(x-z)^{2}+\rho^{2}},
$$

where $z_{\mu}$ and $\rho$ are five free parameters associated with translation invariance and scale invariance. The coefficients $\eta$ are studied in the Appendix. Conjugate to (2.5) we have its mirror image, described by the coefficients $\bar{\eta}$ (see Appendix).

Now these solutions form a local extremum of our functional integrand, and therefore it makes sense to consider separately that contribution to $W$ in (2.4) that is obtained through a new perturbation expansion around these new solutions, taking the integrand there to be approximately Gaussian. The fields $\Phi, \phi$, and $\psi$ all remain infinitesimal so that their mutual interactions may be neglected in the first approximation. Of course, we must also integrate over the values of $z_{\mu}$ and $\rho$. This will be done by means of the collective-coordinate formalism. ${ }^{5}$ One writes

$$
A_{\mu}^{a}=A_{\mu}^{a \mathrm{cl}}+A_{\mu}^{a \mathrm{qu}},
$$

and those values of $A^{\text {qu }}$ that correspond to translations or dilatations are replaced by collective co- ordinates.

The integrand in (2.4) now becomes

$$
\begin{gathered}
\mathcal{L}\left(A^{\mathrm{cl}}\right)-\frac{1}{2}\left(D_{\mu} A_{\nu}^{\mathrm{qu}}\right)^{2}+\frac{1}{2}\left(D_{\mu} A_{\mu}^{\mathrm{qu}}\right)^{2}-g A_{\nu}^{a \mathrm{qu}} \epsilon_{a b c} G_{\mu \nu}^{b c l} A_{\mu}^{c \mathrm{qu}} \\
-D_{\mu} \Phi^{*} D_{\mu} \Phi-\bar{\psi} \gamma_{\mu} D_{\mu} \psi+\bar{\psi} \partial \psi-\frac{1}{2} C_{1}{ }^{2}+\mathcal{L}_{1}^{\text {ghost }} \\
+\mathcal{O}\left(A^{\mathrm{qu}}, \Phi, \psi\right)^{3}
\end{gathered}
$$

where

$$
\begin{aligned}
S^{\mathrm{cl}} & =\int \mathcal{L}\left(A^{\mathrm{c} 1}\right) d^{4} x \\
& =-8 \pi^{2} / g^{2},
\end{aligned}
$$

and the "covariant derivative" $D_{\mu}$ only contains the background field $A_{\mu}^{\mathrm{cl}}$, for instance:

$$
D_{\mu} A_{\nu}^{a \mathrm{qu}}=\partial_{\mu} A_{\nu}^{a \mathrm{qu}}+g \epsilon_{a b c} A_{\mu}^{b c 1} A_{\nu}^{c \mathrm{qu}},
$$

etc.

We abbreviate the integral over (2.7) by

$$
S^{\mathrm{c} 1}-\frac{1}{2} A^{\mathrm{qu}} \mathfrak{M}_{A} A^{\mathrm{qu}}+\bar{\psi} \mathfrak{M}_{\psi} \psi-\Phi * \mathfrak{M}_{\Phi} \Phi-\phi * \mathfrak{M}_{\mathrm{gh}} \phi,
$$

where the last term describes the Faddeev-Popov ghost. Thus, expression (2.4) is (ignoring temporarily the collective coordinates, and certain factors $\sqrt{\pi}$ from the Gaussian integration; see Sec. IX)

$$
\begin{aligned}
W= & \exp \left(-8 \pi^{2} / g^{2}\right)\left(\operatorname{det} \mathfrak{M}_{A}\right)^{-1 / 2} \operatorname{det} \mathfrak{M}_{\psi}\left(\operatorname{det} \mathfrak{M}_{\Phi}\right)^{-1} \\
& \times \operatorname{det} \mathfrak{M}_{\mathrm{gh}} .
\end{aligned}
$$

The determinants will be computed by diagonalization:

$$
\mathfrak{M}_{i} \psi=E_{i} \psi,
$$

after which we multiply all eigenvalues $E$. Since there are infinitely many very large eigenvalues, this infinite product diverges very badly. There are two procedures that will make it converge:

(i) The vacuum-to-vacuum amplitude in the absence of sources must be normalized to 1 , so that the vacuum state has norm 1 . This implies that $W$ must be divided by the same expression with $A^{\mathrm{cl}}=0$.

(ii) We must regularize and renormalize. The dimensional procedure is not available here because the four-dimensionality of the classical solution is crucial. We will use the so-called background Pauli-Villars regulators (Secs. IV and V).

Taking a closer look at the eigenvalue equations (2.12) as they follow from (2.7), we notice that the background field in there gives rise to couplings between spin, isospin, and (the four-dimensional equivalent of) orbital angular momentum, through the coefficients $\eta_{a \mu \nu}$ in (2.5). Now these couplings 
simplify enormously if we go over to a new gauge that explicitly depends on the background field ${ }^{6}$

$$
\begin{aligned}
C_{4}^{a}\left(A^{\mathrm{qu}}\right) & =D_{\mu} A_{\mu}^{a \mathrm{qu}} \\
& =\partial_{\mu} A_{\mu}^{a \mathrm{qu}}+g \epsilon_{a b c} A_{\mu}^{b \mathrm{cl}} A_{\mu}^{c \mathrm{qu}} .
\end{aligned}
$$

Thus the third and eighth terms in (2.7) cancel.

This choice of gauge will lead to one complication, to be discussed in Sec. XI: The gauge for the vacuum-to-vacuum amplitude in the absence of sources, used for normalization, in the region $A_{\mu} \sim 0$, is usually invariant under global isospin rotations, but the classical solution (2.5) and the gauge (2.13) are not. Associated with this will be three spurious zero eigenvalues of $\mathfrak{M}_{A}$ that cannot be directly associated with global isospin rotations. The question is resolved in Sec. XI by careful comparison of the gauge $C_{1}$ with $C_{4}$ and some intermediate choices of gauge.

There will be five other zero eigenvalues of $\mathfrak{M}_{A}$ that of course must not be inserted in the product of eigenvalues directly, since they would render expression (2.11) infinite. They exactly correspond to the infinitesimal translations and dilatations of the classical solution, and, as discussed before, must be replaced by the corresponding collective coordinates (Secs. IX and X).

The matrices $\mathfrak{N}$ are now (ignoring temporarily the fermion source)

$$
\begin{aligned}
& \mathfrak{M}_{A} A_{\mu}^{a \mathrm{qu}}=-D^{2} A_{\mu}^{a \mathrm{qu}}-2 g \epsilon_{a b c} G_{\mu \nu}^{b c 1} A_{\nu}^{c \mathrm{qu}}, \\
& -\mathfrak{M}_{\psi}{ }^{2} \psi=-D^{2} \psi+\frac{1}{4} i \tau^{a} G_{\mu \nu}^{a c 1} \gamma_{\mu} \gamma_{\nu} \psi, \\
& \mathfrak{M}_{\Phi} \Phi=-D^{2} \Phi, \\
& \mathfrak{M}_{\mathrm{gh}} \phi=-D^{2} \phi .
\end{aligned}
$$

In order to substitute the classical solution (2.5) with $z=0$ and $\rho=1$ (generalization to other $z$ and $\rho$ will be straightforward), we introduce the spacetime operators

$$
\begin{aligned}
& L_{1}^{a}=-\frac{1}{2} i \eta_{a \mu \nu} x^{\mu} \frac{\partial}{\partial x^{\nu}}, \\
& L_{2}^{a}=-\frac{1}{2} i \bar{\eta}_{a \mu \nu} x^{\mu} \frac{\partial}{\partial x^{\nu}},
\end{aligned}
$$

with

$$
\begin{aligned}
& {\left[L_{p}^{a}, L_{q}^{b}\right]=i \delta_{p q} \epsilon_{a b c} L_{p}^{c},} \\
& L^{2}=L_{1}^{2}=L_{2}^{2}=-\frac{1}{8}\left(x_{\mu} \partial_{\nu}-x_{\nu} \partial_{\mu}\right)^{2} .
\end{aligned}
$$

They represent rotations in the two invariant $\mathrm{SU}(2)$ subgroups of the rotation group $\mathrm{SO}(4)$.

Isospin rotations will be generated by the operators $T^{a}$ for the scalars, $T^{a}=\frac{1}{2} \tau^{a}$ for the spinors, and $T^{b} A_{\mu}^{a}=i \epsilon_{a b c} A_{\mu}^{c}$ for the vectors. Then

$$
D^{2}=\left(\frac{\partial}{\partial r}\right)^{2}+\frac{3}{r} \frac{\partial}{\partial r}-\frac{4}{r^{2}} L^{2}-\frac{8}{r^{2}+1} T \cdot L_{1}-\frac{4 r^{2}}{\left(r^{2}+1\right)^{2}} T^{2},
$$

where $r^{2}=(x-z)^{2}$. This clearly displays the isospin-orbit coupling.

The vector and spinor fields also have a spinisospin coupling. For the spinors we define the spin operators

$$
\begin{aligned}
& S_{1}^{a} \psi=-\frac{1}{8} i \eta_{a \mu \nu} \gamma_{\mu} \gamma_{\nu} \psi, \\
& S_{2}^{a} \psi=-\frac{1}{8} i \bar{\eta}_{a \mu \nu} \gamma_{\mu} \gamma_{\nu} \psi,
\end{aligned}
$$

satisfying

$$
\left[S_{p}^{a}, S_{q}^{b}\right]=i \delta_{p q} \epsilon_{a b c} S_{p}^{c}
$$

and

$$
\begin{aligned}
& S_{1}{ }^{2}=\frac{3}{4} \frac{1-\gamma_{5}}{2}, \\
& S_{2}{ }^{2}=\frac{3}{4} \frac{1+\gamma_{5}}{2} .
\end{aligned}
$$

For the vector fields we define

$$
\begin{aligned}
& S_{1}^{a} A_{\mu}^{\mathrm{qu}}=-\frac{1}{2} i \eta_{a \mu \nu} A_{\nu}^{\mathrm{qu}}, \\
& S_{2}^{a} A_{\mu}^{\mathrm{qu}}=-\frac{1}{2} i \bar{\eta}_{a \mu \nu} A_{\nu}^{\mathrm{qu}}, \\
& S_{1}{ }^{2}=S_{2}{ }^{2}=\frac{3}{4} .
\end{aligned}
$$

For the scalar fields $\vec{S}_{1}=\vec{S}_{2}=0$. Thus right- and left-handed spinors are $\left(\frac{1}{2}, 0\right)$ and $\left(0, \frac{1}{2}\right)$ representations of $\mathrm{SO}(4)$, and vectors are $\left(\frac{1}{2}, \frac{1}{2}\right)$ representations. Scalars of course are $(0,0)$ representations.

In terms of the operators $S$ and $T$, the spinisospin couplings turn out to be universal for all particles. Substituting the classical value for $G_{\mu \nu}^{a c 1}$ in (2.14) we find

$$
\begin{aligned}
\mathfrak{M}= & -\left(\frac{\partial}{\partial r}\right)^{2}-\frac{3}{r} \frac{\partial}{\partial r}+\frac{4}{r^{2}} L^{2}+\frac{8}{1+r^{2}} T \cdot L_{1} \\
& +\frac{4 r^{2}}{\left(1+r^{2}\right)^{2}} T^{2}+\frac{16}{\left(1+r^{2}\right)^{2}} T \cdot S_{1}
\end{aligned}
$$

with $\mathfrak{M}=\mathfrak{M}_{A}$ or $-\mathfrak{M}_{\psi}^{2}$ or $\mathfrak{M}_{\Phi}$ or $\mathfrak{M}_{\phi}$.

Observe the absence of spin-orbit and isospinorbit couplings that contain $x_{\mu}$ or $\partial / \partial x_{\mu}$ explicitly. It all goes via the orbital angular momentum operator $L_{1}$ and that implies that $L^{2}$ commutes with $\Re$. This would not be so in other gauges. Further, 97 commutes with $\vec{J}_{1}=\overrightarrow{\mathrm{L}}_{1}+\overrightarrow{\mathrm{S}}_{1}+\overrightarrow{\mathrm{T}}$ and $\overrightarrow{\mathrm{L}}_{2}$ and $\overrightarrow{\mathrm{S}}_{2}$. Eigenvectors of $\mathfrak{M}$ can thus be characterized by the quantum numbers 
$s_{1}$ and $s_{2}$ (both either 0 or $\frac{1}{2}$ ),

$t$ (total isospin, arbitrary for the scalars, $\frac{1}{2}$ for the spinors, 1 for the vector and the ghost),

$l=0, \frac{1}{2}, 1, \ldots$,

$j_{1}=l-s_{1}-t, l-s_{1}-t+1, \ldots, l+s_{1}+t$, as long as $j_{1} \geqslant 0$,

$j_{1}{ }^{3}=-j_{1}, \ldots,+j_{1}$,

$s_{2}{ }^{3}=-s_{2}, \ldots,+s_{2}$,

$l_{2}^{3}=-l, \ldots,+l$.

For normalization we need the corresponding operator $\mathbb{M}$ for the case that the background field is zero:

$$
\mathfrak{M} \mathbb{0}_{0}=-\left(\frac{\partial}{\partial r}\right)^{2}-\frac{3}{r} \frac{\partial}{\partial r}+\frac{4}{r^{2}} L^{2} .
$$

\section{DETERMINANTS AND PHASE SHIFTS}

The eigenvalue equation (2.12) with $\mathfrak{M}$ as in (2.22) differs in no essential way from an ordinary Schrödinger scattering problem. In this section we show the relation between the corresponding scattering matrix and the desired determinant.

Temporarily, we put the system in a large spherical box with radius $R$. At the edge we have some boundary condition: either $\Psi(R)=0$, or $\Psi^{\prime}(R)=0$ (or a linear combination thereof). Here $\Psi$ stands for any of the scalar, spinor, or vector fields. In the case $\Psi^{\prime}(R)=0$ the vacuum operator $\mathfrak{M}_{0}$ has a zero eigenvalue corresponding to $\Psi$ = constant, and also the lowest eigenvalue of $\mathfrak{T}$ may go to zero more rapidly than $1 / R^{2}$ when $R \rightarrow \infty$. Such eigenvalues have to be considered separately (negative eigenvalues can be proved not to exist).

We here consider all other eigenvalues of $\mathfrak{T}$. They approach the ones of $\mathfrak{M}_{0}$ if $R \rightarrow \infty$. We wish to compute the product

$$
\prod_{n=1}^{\infty} \frac{E(n)}{E_{0}(n)}
$$

The scattering matrix $S(k)=e^{2 i \eta(k)}$ will be defined by comparing the solution of

$$
\mathfrak{M} \Psi=k^{2} \Psi
$$

with

$$
\mathfrak{T}_{0} \Psi_{0}=k^{2} \Psi_{0}
$$

both with boundary condition $\Psi \rightarrow C r^{2 t}$ at $r=0$. Let

$$
\begin{aligned}
\Psi_{0}(r) & \sim C r^{-3 / 2}\left(e^{-i k(r+a)}+e^{+i k(r+a)}\right) \\
& =2 C r^{-3 / 2} \cos k(r+a) \text { for large } r
\end{aligned}
$$

and

$$
\begin{aligned}
\Psi(r) & \propto C r^{-3 / 2}\left[e^{-i k(r+a)}+S(k) e^{i k(r+a)}\right] \\
& =2 C^{\prime} r^{-3 / 2} \cos [k(r+a)+\eta(k)]
\end{aligned}
$$

If we require at $r=R$ the same boundary condition for $\Psi_{0}$ then we must solve

$$
k(n)(R+a)+\eta(k(n))=k_{0}(n)(R+a),
$$

thus

$$
\frac{k(n)}{k_{0}(n)} \rightarrow 1-\frac{\eta(k(n))}{(R+a) k(n)} .
$$

The level distance $\Delta k=k(n+1)-k(n)$ is in both cases, asymptotically for large $R$,

$$
\Delta k=\frac{\pi}{R}+O\left(\frac{1}{R^{2}}\right) .
$$

We find that

$$
\begin{aligned}
\prod_{n=1}^{\infty} \frac{E(n)}{E_{0}(n)} & =\exp \left\{2 \sum_{1}^{\infty} \ln \left[k(n) / k_{0}(n)\right]\right\} \\
& =\exp \left\{2 \frac{R}{\pi} \sum_{1}^{\infty} \Delta k\left[-\frac{\eta(k)}{R k}+O\left(\frac{1}{R^{2}}\right)\right]\right\} \\
& \rightarrow \exp \left[-\frac{2}{\pi} \int_{0}^{\infty} \frac{\eta(k)}{k} d k\right]
\end{aligned}
$$

provided that the integral converges at both ends.

At $k \rightarrow 0$ the integral (3.9) converges provided that the interaction potential decreases faster than $1 / r^{2}$ as $r \rightarrow \infty$; at $k \rightarrow \infty$ the integral converges if the interaction potential is less singular than $1 / r^{2}$ as $r \rightarrow 0$. The latter condition is satisfied if we compare $\mathfrak{N}$ and $\mathfrak{M}_{0}$ at the same values for the quantum number $l$; the first condition is satisfied if $\left(L_{1}+T\right)^{2}$ for the interacting matrix is set equal to $L^{2}$ for the vacuum matrix. If we consider the combined effect of all values for $L^{2}$ and $\left(L_{1}+T\right)^{2}$ both for the vacuum and for the interacting case then we can split the integral (3.9) somewhere in the middle, and combine the $k \rightarrow \infty$ parts, so that we get convergence everywhere. 
An easier way to get convergence is to regularize:

$$
\begin{aligned}
& \prod_{n=1}^{\infty} \frac{E(n)\left[E_{0}(n)+M^{2}\right]}{E_{0}(n)\left[E(n)+M^{2}\right]} \\
& \rightarrow \exp \left[-\frac{1}{\pi} \int_{0}^{\infty} \frac{\eta(k)}{k} \frac{M^{2}}{\left(k^{2}+M^{2}\right)} d k\right] .
\end{aligned}
$$

Regulators will be introduced anyhow, so we will not encounter difficulties due to no convergence of the integral in (3.9).

\section{ELIMINATION OF THE SPIN DEPENDENCE}

In Eq. (2.22) the operators $T \cdot L_{1}$ and $T \cdot S_{1}$ do not commute. Only in the case that

$$
\left|j_{1}-l\right|=s+t
$$

(as defined in 2.23) do they simultaneously diagonalize. If

$$
\left|j_{1}-l\right|<s+t
$$

and

$$
j_{1} \neq 0, \quad l \neq 0, \quad s=\frac{1}{2}, \quad t \neq 0,
$$

then we have a set of coupled differential equations for two dependent variables.

In any other case there would be no hope of solving this set of equations analytically, but here we can make use of a unique property of the equation

$$
\Re \Psi=E \Psi,
$$

which enables us to diagonalize it completely. If $s_{1}=0$ the equation could describe a left-handed fermion with isospin $t$ :

$$
\begin{aligned}
& \mathfrak{T} \psi=-(\gamma \cdot D)^{2} \psi=E \psi, \\
& \gamma_{5} \psi=+\psi .
\end{aligned}
$$

But then we can define, if $E \neq 0$,

$$
\psi^{\prime}=\gamma \cdot D \psi
$$

with

$$
\begin{aligned}
& \Re \psi^{\prime}=E \psi^{\prime}, \\
& \gamma_{5} \psi^{\prime}=-\psi^{\prime}
\end{aligned}
$$

Now $\psi^{\prime}$ has $s_{1}^{\prime}=\frac{1}{2}$, and hence we found a solution for the set of coupled equations with $s_{1}^{\prime}=\frac{1}{2}$ from a solution of the simpler equation with $s_{1}=0$. The operater $\gamma \cdot D$ in Eq. (4.3) does not commute with $L^{2}$, so if $\psi$ has a given set of quantum numbers $l, j_{1}, t$ then $\psi^{\prime}$ is a superposition of a state with $l^{\prime}$ $=l+\frac{1}{2}$ and one with $l^{\prime}=l-\frac{1}{2}$. Now $\mathfrak{M}$ does commute with $L^{2}$, so if we project out the state with $l^{\prime}=l+\frac{1}{2}$ or $l^{\prime}=l-\frac{1}{2}$ then we get a new solution in both cases. Thus one solution with $s_{1}=0$ and quantum numbers $l, j_{1}, t$ generates two solutions with $s_{1}^{\prime}=\frac{1}{2}, l^{\prime}=l \pm \frac{1}{2}$, $j_{1}^{\prime}=j_{1}, t^{\prime}=t$. In terms of the operators $L, S$, and $T$ the new solutions to the coupled equations can be expressed in terms of the $S=0$ solutions as follows:

$$
\begin{aligned}
& L_{a}^{\prime}+S_{a}^{\prime}=L_{a}, \quad l^{\prime}=l \pm \frac{1}{2}, \\
& T^{\prime}=T, \quad J^{\prime}=J, \\
& \Psi^{\prime}=\left[\frac{1}{r}\left(2 L^{2}-2 L^{\prime 2}+\frac{3}{2}\right)+\frac{4 r}{1+r^{2}} S_{1}^{\prime} \cdot T\right] \Psi+\frac{\partial}{\partial r} \Psi .
\end{aligned}
$$

It is easy to check explicitly that if $\Psi$ satisfies (2.22) with $S_{1}=0$, then the two wave functions $\Psi^{\prime}$ both satisfy (2.22) when the operators $L, S, T$ are replaced by the primed ones.

Asymptotically, for large $r, \Psi^{\prime}=(\partial / \partial r) \Psi$, and hence the phase shift $\eta(k)$ is the same for the primed case as for the original case. Consequently, the integral over the phase shifts as it occurs in (3.9) is the same for spinor and vector fields (with $s_{1}=\frac{1}{2}$ ) as it is for scalar fields (with $s_{1}=0$ ).

The above procedure becomes more delicate if $E=0$. Indeed, although scalar fields can easily be seen to have no zero-eigenvalue modes, spinor and vector fields do have them. In conclusion, the nonzero eigenvalues for the vector and spinor modes are the same as for the scalar modes, but the zero eigenvectors are different.

In the following sections we compute the universal value for the product. Note that also the regularized expressions (3.10) are equivalent because the $\eta(k)$ match for all $k$. The regulator of Eq. (3.10) corresponds to new fields with Lagrangians

$$
\begin{aligned}
& \mathscr{L}=-\frac{1}{2}\left(D_{\mu} B_{\nu}\right)^{2}-\frac{1}{2} M^{2} B_{\nu}{ }^{2}-g B_{\nu}^{a} \epsilon_{a b c} G_{\mu \nu}^{b} B_{\mu}^{c} \\
& \quad \text { for vectors, } \\
& \mathscr{L}=\bar{\chi}\left[-(\gamma \cdot D)^{2}-M^{2}\right] \chi \text { for spinors },
\end{aligned}
$$

and

$$
\mathcal{L}=-\left(D_{\mu} \xi\right) * D_{\mu} \xi-M^{2} \xi^{*} \xi \text { for scalars . }
$$

Within the background field procedure it is obvious that such regulator fields make the one-loop amplitudes finite. Later (Sec. XIII) we will make the link with the more conventional dimensional regulators.

\section{A NEW EIGENVALUE EQUATION AND NEW REGULATORS}

As stated in the Introduction, the solutions to the equations $\mathfrak{T} \Psi=E \Psi$ even in the scalar case cannot be expressed in terms of simple elementary functions. But eventually we only need det $\Re /$ $\operatorname{det} T_{0}$, and this can be obtained in another way. 
We write

$$
\begin{aligned}
& V=\frac{1}{4}\left(1+r^{2}\right) \mathfrak{M}\left(1+r^{2}\right), \\
& V_{0}=\frac{1}{4}\left(1+r^{2}\right) \mathfrak{M}_{0}\left(1+r^{2}\right),
\end{aligned}
$$

and, formally,

$$
\operatorname{det}\left(\mathfrak{M} / \mathfrak{M}_{0}\right)=\operatorname{det}\left(V / V_{0}\right) .
$$

The equation

$$
V \Psi=\lambda \Psi
$$

corresponds to the expression

$$
\left[\left(\frac{\partial}{\partial r}\right)^{2}+\frac{3}{r} \frac{\partial}{\partial r}-\frac{4}{r^{2}} L^{2}-\frac{4}{1+r^{2}}\left(J_{1}^{2}-L^{2}\right)+\frac{4\left(T^{2}+\lambda\right)}{\left(1+r^{2}\right)^{2}}\right] \Psi=0 .
$$

Write

$$
\begin{aligned}
& x=\frac{1}{1+r^{2}}, \\
& \Psi=r^{2 l}\left(1+r^{2}\right)^{-l-j_{1}-1} \Phi(x),
\end{aligned}
$$

then

$$
\left\{\left(\frac{\partial}{\partial x}\right)^{2}+\left[\frac{2\left(j_{1}+1\right)}{x}-\frac{2(l+1)}{1-x}\right] \frac{\partial}{\partial x}+\frac{1}{x(1-x)}\left[T^{2}+\lambda-\left(l+j_{1}+1\right)\left(l+j_{1}+2\right)\right]\right\} \Phi=0 .
$$

This is a hypergeometric equation. The physical region is $1 /\left(1+R^{2}\right)<x \leqslant 1$. In the Hilbert space of square-integ rable wave functions the spectrum is now discrete, which implies that we can safely take the limit $R \rightarrow \infty$. The solutions for $\Phi$ are just polynomials:

$$
\begin{aligned}
& \Phi(x)=\sum_{\nu=0}^{\infty} a_{\nu} x^{\nu}, \\
& a_{\nu+1}=a_{\nu} \frac{(\nu-n)\left(\nu+n+2 l+2 j_{1}+3\right)}{(\nu+1)\left(\nu+2 j_{1}+2\right)},
\end{aligned}
$$

where $n$ is defined by

$$
\left(n+l+j_{1}+1\right)\left(n+l+j_{1}+2\right)=T^{2}+\lambda .
$$

If $n=$ integer $\geqslant 0$ then the series (5.7) breaks off. Otherwise $\Phi$ is not square-integrable. So we find the eigenvalues

$$
\begin{aligned}
& \lambda_{n}=\left(n+l+j_{1}+1-t\right)\left(n+l+j_{1}+2+t\right), \\
& n=0,1,2, \ldots, \quad T^{2}=t(t+1) .
\end{aligned}
$$

The vacuum case, $V_{0} \Psi=\lambda_{0} \Psi$, is solved by the same equation, but with $j_{1}=l, t=0$.

The product of these eigenvalues, even when divided by the vacuum values, still badly diverges so we must regularize. The regulators of Sec. IV are not very attractive here because they spoil the hypergeometric nature of the equations. More convenient here is a set of regulator fields with masses that all depend on space-time in a certain way. They are given by the Lagrangians (4.7)-(4.9) but with $M^{2}$ replaced by

$$
\frac{4 M^{2}}{\left(1+r^{2}\right)^{2}}
$$

We choose $M^{2}$ here so large that anywhere near the origin the regulator is heavy. Far from the origin the classical solution is expected to be close enough to the real vacuum, so that there the details of the regulators are irrelevant.

Of course the regulator procedure affects the definition of the subtracted coupling constant. In Sec. VII we link the regulator (5.11) with the more acceptable one of Sec. IV, and in Sec. XIII we make the link with the dimensional regulator.

The eigenvalues of the regulator are

$$
\lambda_{n}^{M}=\left(n+l+j_{1}+1-t\right)\left(n+l+j_{1}+2+t\right)+M^{2} .
$$

The regulators $M_{i}$ with $i=1, \ldots, R$ are as usual of alternating metric $e_{i}= \pm 1$. Consequently, det9l is replaced by

$$
(\operatorname{det} \Re) \prod_{i=1}^{R}\left(\operatorname{det} \pi_{i}\right)^{e_{i}}
$$

This converges rapidly if

$$
\begin{aligned}
& \sum_{1}^{R} e_{i}=-1 \\
& \sum_{i}^{R} e_{i} M_{i}=0 \\
& \sum_{1}^{R} e_{i} M_{i}^{2}=0, \ldots,
\end{aligned}
$$

and

$$
\sum_{i}^{R} e_{i} \ln M_{i} \equiv-\ln M=\text { finite. }
$$

Let $i=0$ denote the physical field, then

$$
e_{0}=1, \quad M_{0}=0, \quad \sum_{i=0}^{R} e_{i}=0, \text { etc. }
$$




\section{THE REGULARIZED PRODUCT OF THE NONVANISHING EIGENVALUES}

We now consider the logarithm of the regularized product of the nonvanishing eigenvalues, for a scalar field with total isospin $t$ :

$$
\ln \Pi(t)=\sum_{i=0}^{R} e_{i} \sum \ln \lambda^{M_{i}}
$$

with

$$
\lambda^{M_{i}}=\left(n+l+j_{1}+1-t\right)\left(n+l+j_{1}+2+t\right)+M_{i}^{2}
$$

(we imply that $e_{0}=1$ and $M_{0}=0$ ). The summation goes over the values of all quantum numbers. Now for given $n, l, j_{1}$ the degeneracy is $\left(2 j_{1}+1\right)\left(2 j_{2}+1\right)$ $=\left(2 j_{1}+1\right)(2 l+1)$. The values of $l, j_{1}$, and $n$ are restricted by

$$
\begin{aligned}
& \sigma \equiv l+j_{1}-t \geq 0, \\
& \tau \equiv j_{1}-l+t \geq 0, \quad \tau \leq 2 t, \quad n \geq 0 .
\end{aligned}
$$

[Later we will divide $\Pi(t)$ by the vacuum value $\Pi_{0}(t)$, which is obtained by the same formulas as above and the following, but with $t$ replaced by zero, and the degeneracy will be $(2 t+1)(2 l+1)^{2}$.]

We go over to the variables $\sigma$ and $\tau$ as given by (6.3) and $s$ with

$$
s=n+l+j_{1}+\frac{3}{2}, \quad s \geq t+\sigma+\frac{3}{2} .
$$

We find that

$$
\begin{aligned}
\ln \Pi(t)=\sum_{s=t+3 / 2}^{\infty} \sum_{i} e_{i} \sum_{\tau=0}^{2 t} \sum_{\sigma=0}^{s-t-3 / 2} & (\sigma+\tau+1)(2 t+\sigma-\tau+1) \\
& \times \ln \left[s^{2}+M_{i}^{2}-\left(t+\frac{1}{2}\right)^{2}\right] .
\end{aligned}
$$

The summation over $\sigma$ and $\tau$ gives

$$
\begin{aligned}
\ln \Pi(t)=\frac{2 t+1}{3} \sum_{s=t+3 / 2}^{\infty} \sum_{i} & e_{i}\left[s^{3}-s\left(t+\frac{1}{2}\right)^{2}\right] \\
& \times \ln \left[s^{2}+M_{i}{ }^{2}-\left(t+\frac{1}{2}\right)^{2}\right]
\end{aligned}
$$

The vacuum value $\Pi_{0}(t)$ is obtained from (6.6) by replacing $t$ with zero and adding an additional multiplicity $2 t+1$, thus

$$
\begin{aligned}
\ln \Pi_{0}(t)=\frac{2 t+1}{3} \sum_{s=3 / 2}^{\infty} \sum_{i} & e_{i}\left(s^{3}-\frac{1}{4} s\right) \\
& \times \ln \left(s^{2}+M_{i}^{2}-\frac{1}{4}\right) .
\end{aligned}
$$

Now we interchange the summation over $s$ and $i$, letting first $s$ go from $t+\frac{3}{2}$ to $\Lambda$ and taking $\Lambda \rightarrow \infty$ in the end. We get

$$
\ln \left[\Pi(t) / \Pi_{0}(t)\right]=\frac{2 t+1}{3} \sum_{i} e_{i}\left(A^{M i}\left(t+\frac{1}{2}\right)-A^{M i}\left(\frac{1}{2}\right)\right)
$$

with

$$
A^{M i}(\phi)=\sum_{s=0+1}^{\Lambda}\left(s^{3}-s \phi^{2}\right) \ln \left(s^{2}+M_{i}{ }^{2}-\phi^{2}\right) .
$$

Let us first consider the regulator contribution. Then $M$ is large. We may consider the logarithm as a slowly varying function, and approximate the summation by means of the Euler-Maclaurin formula,

$$
\begin{aligned}
\sum_{s=p+1}^{\Lambda} f(s)=\int_{p}^{\Lambda} f(x) d x+ & {\left[\frac{1}{2} f(x)+\frac{1}{12} f^{\prime}(x)\right.} \\
& \left.-\frac{1}{720} f^{\prime \prime \prime}(x) \cdots\right]\left.\right|_{p} ^{\Lambda},
\end{aligned}
$$

and we obtain

$$
\begin{aligned}
A^{M}(\phi)= & \operatorname{indep}(\phi)+\phi^{2}\left(-\frac{1}{2} M^{2}-\Lambda^{2} \ln \Lambda-\Lambda \ln \Lambda\right. \\
& \left.-\frac{1}{2} \Lambda-\frac{1}{6} \ln \Lambda-\frac{1}{4}-\frac{1}{6} \ln M^{2}\right) \\
& +\frac{1}{4} \phi^{4}(2 \ln \Lambda+1)+O\left(\frac{1}{M^{2}}\right)+O\left(\frac{1}{\Lambda}\right) .
\end{aligned}
$$

The first term stands for an array of expressions, all independent of $\phi$, and is not needed because it cancels out in Eq. (6.8).

For $A^{0}(\phi)$ the series (6.10) will not converge at $x=p$ so it cannot be used. After some purely algebraic manipulations we find

$$
\begin{aligned}
A^{0}(\phi)= & \sum_{s=\phi+1}^{\Lambda} s(s+\phi)(s-\phi)[\ln (s+\phi)+\ln (s-\phi)] \\
= & \operatorname{indep}(\phi)+4 \phi^{2} \sum_{s=1}^{\Lambda} s \ln s+\sum_{s=1}^{2 \phi} s(2 \phi-s)(s-\phi) \ln s \\
& +\phi^{2}\left(-3 \Lambda^{2}-3 \Lambda-\frac{1}{2}\right) \ln \Lambda+\phi^{2}\left(\Lambda^{2}-\frac{1}{2} \Lambda-\frac{7}{12}\right)+\phi^{4}\left(\frac{1}{2} \ln \Lambda-\frac{1}{12}\right)+O\left(\frac{1}{\Lambda}\right) .
\end{aligned}
$$


Now we insert (6.11) and (6.12) into (6.8):

$$
\begin{aligned}
\ln \left[\Pi(t) / \Pi_{0}(t)\right]=\frac{2 t+1}{3} & \left\{\sum_{s=1}^{2 t+1} s(2 t+1-s)\left(s-t-\frac{1}{2}\right) \ln s\right. \\
& \left.+t(t+1)\left[4 \sum_{1}^{\Lambda} s \ln s-2 \Lambda^{2} \ln \Lambda-2 \Lambda \ln \Lambda-\frac{1}{3} \ln \Lambda+\Lambda^{2}+\frac{1}{3} \ln M-\frac{1}{3} t(t+1)-\frac{1}{2}\right]\right\} .
\end{aligned}
$$

We made use of $\sum_{0}^{R} e_{i}=0, \sum_{0}^{R} e_{i} M_{i}^{2}=0, \sum_{1}^{R} e_{i} \ln M_{i}^{2} \equiv-\ln M$. The limit $\Lambda \rightarrow \infty$ exists. Defining

$$
\begin{aligned}
R & =\lim _{\Lambda \rightarrow \infty}\left(\sum_{s=1}^{\Lambda} s \ln s-\frac{1}{2} \Lambda^{2} \ln \Lambda-\frac{1}{2} \Lambda \ln \Lambda-\frac{1}{12} \ln \Lambda+\frac{1}{4} \Lambda^{2}\right) \\
& =0.248754477,
\end{aligned}
$$

we find that

$$
\ln \left[\Pi(t) / \Pi_{0}(t)\right]=\frac{t(t+1)(2 t+1)}{3}\left[\frac{1}{3} \ln M+4 R+\sum_{s=1}^{2 t+1} s(2 t+1-s)\left(s-t-\frac{1}{2}\right) \ln s-\frac{1}{3} t(t+1)-\frac{1}{2}\right] .
$$

$R$ is related to the Riemann zeta function $\zeta(z)$ as follows:

$$
\begin{aligned}
R & =\frac{1}{12}-\zeta^{\prime}(-1) \\
& =\frac{\ln 2 \pi+\gamma}{12}-\frac{\zeta^{\prime}(2)}{2 \pi^{2}} \\
& =\frac{1}{12}(\ln 2 \pi+\gamma)+\frac{1}{2 \pi^{2}} \sum_{s=1}^{\infty} \frac{\ln s}{s^{2}},
\end{aligned}
$$

$\gamma=0.5772156649$ is Euler's constant, and

$$
\begin{aligned}
-\zeta^{\prime}(2) & =\sum \frac{\ln s}{s^{2}} \\
& =0.937548254315844 .
\end{aligned}
$$

\section{THE FIXED MASS REGULATOR}

Equation (6.15) gives the regularized product of all nonvanishing eigenvalues of $\mathfrak{m}$. But the regulator used was a very unsatisfactory one, from a physical point of view, because the regulator mass $\mu$ depends on space-time:

$$
\mu^{2}=\frac{4 M^{2}}{\left(1+r^{2}\right)^{2}} \text {. }
$$

This $\mu$ must be interpreted as the subtraction point of the coupling constant $g$. Now $g$ does not occur in $\Pi(t) / \Pi_{0}(t)$, but it does occur in the expression for the total action for the classical solution, and as we emphasized in the Introduction, any change in the subtraction procedure is important. The problem here is that we wish to make a spacetime-dependent change in the subtraction point, from $\mu$ to a fixed $\mu_{0}$. We solve that in the following way.

The effect of a change in the regulator mass can be absorbed by a counterterm in the Lagrangian, and hence is local in space-time. So we expect that, if we make a space-time-dependent change in the regulator mass, then this change can be absorbed by a space-time-dependent counterterm. Moreover, since our regulators are both gaugeinvariant, this counterterm is gauge invariant. For space-time-independent regulators, this counterterm can be computed by totally conventional methods:

$$
\Delta \mathfrak{L}=\frac{-g^{2}}{32 \pi^{2}} G_{\mu \nu} G_{\mu \nu} \times \frac{1}{9} t(t+1)(2 t+1) \ln \left(\mu / \mu_{0}\right) .
$$

From locality we deduce that the same formula must also be true for space-time-dependent regulator mass $\mu(x)$, simply because no other gaugeinvariant, local expressions of the same dimensionality exist. Inserting the classical value for $G_{\mu \nu}$,

$$
G_{\mu \nu}^{a \mathrm{c} 1}=-\frac{4}{g} \frac{\eta_{a \mu \nu}}{\left(1+r^{2}\right)^{2}},
$$

and expression (7.1) for $\mu$, we get

$$
\begin{aligned}
\Delta S^{\mathrm{c} 1}= & \int \Delta \mathcal{L} d^{4} x \\
= & \frac{16 \times 12 \pi^{2}}{32 \pi^{2} \times 9} t(t+1)(2 t+1) \\
& \times \int_{0}^{\infty} \frac{r^{2} d r^{2}}{\left(1+r^{2}\right)^{4}} \ln \frac{\mu_{0}}{2 M}\left(1+r^{2}\right) \\
= & \frac{2}{3} t(t+1)(2 t+1)\left(\frac{1}{6} \ln \frac{\mu_{0}}{2 M}+\frac{5}{36}\right) .
\end{aligned}
$$


In the expression

$$
\frac{\Pi_{0}(t)}{\Pi(t)} S^{\mathrm{c} 1},
$$

with $\Pi(t) / \Pi_{0}(t)$ as computed in (6.15), we must correct $S^{\mathrm{c} 1}$ with the above $\Delta S^{\mathrm{c} 1}$, in order to get the corresponding expression with $g$ subtracted with fixed mass regulators, as defined in (4.9). The regulator masses $M_{i}$ in there must be such that

$$
\sum_{i}^{R} e_{i} \ln M_{i}=-\ln \mu_{0} .
$$

Expression (7.4) must be added to (6.15). Thus we get

$$
\ln \left[\Pi(t) / \Pi_{0}(t)\right]=\frac{t(t+1)(2 t+1)}{3}\left[\frac{1}{3} \ln \frac{\mu_{0}}{2}+4 R+\sum_{s=1}^{2 t+1} s(2 t+1-s)\left(s-t-\frac{1}{2}\right) \ln s-\frac{1}{3} t(t+1)-\frac{2}{9}\right] .
$$

We note that the coefficient of the regulator term in $(6.15)$ has the correct value. It matches the coefficient of (7.2) that has been computed independently. The regulator in this expression, (7.6), is the same as the one used in (3.10), and so we can use the result of Sec. IV to do the spinor and vector fields.

In Sec. IV we proved that the nonzero eigenvalues for vector and spinor fields are the same as for scalar fields, but we must take some multiplicity factors into account. Equation (7.6) holds for one complex scalar multiplet with isospin $t$. Fields with integer isospin may be real and then we have to multiply by $\frac{1}{2}$. The vector field has four components but is real, and hence its value for $\ln \left(\Pi / \Pi_{0}\right)$ is twice expression (7.6), with $t=1$. The complex Faddeev-Popov ghost has Fermi statistics and contributes with one unit, but opposite sign. Thus, altogether, the vector field contributes just like one complex scalar with $t=1$.

For fermions we must compute $\operatorname{det} \mathfrak{M T}_{\psi}$, but the theorem of Sec. IV applies to $\mathfrak{M}_{\psi}^{2}$. The fermions have four Dirac components. So, altogether, fermions contribute just like two complex scalars, but the sign in $\ln \left(\Pi / \Pi_{0}\right)$ is opposite because of fermi statistics.

The above summarizes in words the complete contribution of all nonzero eigenstates to the functional determinants. But the spinor and vector fields have a few more modes, with $E=0$, and also the regulators have corresponding new modes, with $E=\mu_{0}^{2}$.

\section{THE ZERO EIGENSTATES}

First we consider the vector fields. We have $s_{1}=\frac{1}{2}, t=1$. Careful study of the operator $\mathfrak{M}$, Eq. (2.22), enables us to list the square-intergrable zero eigenstates as follows:

$$
\begin{aligned}
& \text { (i) } j_{1}=\frac{1}{2}, l=0: \quad \Psi=\left(1+r^{2}\right)^{-2} \\
& \left(j_{2}=s_{2}=\frac{1}{2}\right), \\
& \text { multiplicity }=\left(2 j_{1}+1\right)\left(2 j_{2}+1\right)=4 \text {. }
\end{aligned}
$$

$$
\text { (ii) } j_{1}=0, l=\frac{1}{2}: \Psi=r\left(1+r^{2}\right)^{-2} \text {. }
$$

There are two possibilities for the other quantum numbers:

$$
\begin{aligned}
& \text { (a) } j_{2}=0 \text {, multiplicity }=1 \text {, } \\
& \text { (b) } j_{2}=1, \text { multiplicity }=3 \text {. }
\end{aligned}
$$

This completes the set of zero eigenstates. We interpret these as follows. States (i) have $j_{1}=j_{2}$ $=\frac{1}{2}$, that is, the quantum numbers of an infinitesimal translation. The translations are considered in Sec. IX. State (iia) is the only singlet. It will correspond to the infinitesimal dilatation, Sec. X. State (iib) is just an anomaly. It will be discussed in Sec. XI. It is indirectly connected with infinitesimal global isospin rotations.

Spinors have similar sets of eigenstates, but their interpretation will be totally different. If $t=1$, then the eigenstates are essentially the same as the vector ones, but their multiplicity is half of that because $s_{2}=0$. In this paper we limit ourselves to $t=\frac{1}{2}$. Then there is just one zero eigenstate:

$$
j_{1}=0, \quad l=j_{2}=0, \quad \Psi=\left(1+r^{2}\right)^{-3 / 2} .
$$

Its multiplicity is of course $N^{f}$ if there are $N^{f}$ flavors. It leads to an $N^{f}$-fold zero in the amplitude [note that in (2.11) the amplitude is proportional to $\operatorname{det} \mathfrak{M}_{\psi}$ and thus is proportional to the product of the eigenvalues of $\mathfrak{T}_{\psi}$; if we have $N^{f}$ zero eigenvalues then $W$ has an $N^{f}$-fold zero]. But this zero will be removed if we switch on the fermion source $\mathcal{J}$ in the Lagrangian (2.1). In Sec. XIV we will construct the resulting $N^{f}$-point Green's function.

In strong-interaction theories the fermion mass will also remove this zero. The zero eigenstates must also be included in the regulator contributions. From Sec. VII on, our regulator mass is fixed and is essentially equal to $\mu_{0}$. Every zero eigenvector of the operator $\mathfrak{M}$, Eq. (2.22), will be accompanied by a factor $\mu_{0}^{-2}$ for the regulator (a zero eigenvector of $\mathfrak{M T}_{\psi}$ is accompanied by a factor $\left.\mu_{0}^{-1}\right)$. 


\section{COLLECTIVE COORDINATES: 1 . TRANSLATIONS}

Clearly, zero eigenvalues make no sense if they would be included in the products carefully computed in the previous sections. In the case of the vector fields, which we will now discuss, they would render the functional integral infinite because they are in the denominator. It merely means that the integration in those directions is not Gaussian.

Let us first consider the four modes (8.1). The angular dependence and index dependence can be read off from the quantum numbers. Written in full, the mode corresponds to the quantum field fluctuation (with arbitrarily chosen norm):

$$
A_{\mu}^{a \mathrm{qu}}(\nu)=2 \eta_{a \mu \nu}\left(1+r^{2}\right)^{-2}, \quad \nu=1, \ldots, 4 .
$$

This can be seen to be the space-time derivative of the classical solution up to a gauge transformation:

$$
A_{\mu}^{a \mathrm{qu}}(\nu)=-\left.\frac{g}{2} \frac{\partial}{\partial z^{\nu}}\left\{\frac{2 \eta_{a \mu \lambda}(x-z)^{\lambda}}{g\left[1+(x-z)^{2}\right]}\right\}\right|_{z=0}+D_{\mu} \Lambda^{a}(\nu),
$$

with

$$
\Lambda^{a}(\nu)=-\eta_{a \nu \lambda} x^{\lambda}\left(1+x^{2}\right)^{-1} .
$$

The gauge transformation is there because our gauge-fixing term depends on the background field.

If we want to replace the variable $D A^{\mathrm{qu}}$ in this particular zero-mode direction by the collective variables $d z^{\nu}$, then we must insert the corresponding Jacobian factor ${ }^{5}$

$$
\int D A^{\mathrm{qu}} \rightarrow \int \prod_{\nu}\left(\frac{2}{g} d z^{\nu}\right)\left\{\frac{1}{\pi} \int\left[A_{\mu}^{a \mathrm{qu}}(\nu)\right]^{2} d^{4} x\right\}^{1 / 2},
$$

where $A_{\mu}^{a \text { qu }}(\nu)$ is the solution (9.1). The factor $2 / g$ comes from the factor $g / 2$ in (9.2). This way the result is independent of the normalization of $A_{\mu}^{a}$ qu $(\nu)$ of (9.1). The factors $\pi^{-1 / 2}$ arise from the fact that we compare this integral with Gaussian integrals, and in these Gaussian integrals the factors $\sqrt{\pi}$ that go with each eigenvalue had been suppressed previously. We could also have dragged along all factors $\sqrt{\pi}$ at each of the eigenvalues of the matrices $\Re$, and then we would have noticed that the factors $\sqrt{\pi}$ going with the corresponding modes of the regulators, which are still Gaussian, would have been left over. In (9.4) we just include these factors from the beginning.

The norm of the solution (9.1) is

$$
\int A_{\mu}^{a \mathrm{qu}}(\nu) A_{\mu}^{a \mathrm{qu}}(\lambda) d^{4} x=2 \pi^{2} \delta_{\nu \lambda},
$$

so, together with the regulator, these four modes yield the factor

$$
\left(\frac{2}{g}\right)^{4}\left(\frac{\mu_{0}^{2}}{\pi}\right)^{2}\left(2 \pi^{2}\right)^{2} d^{4} z=2^{6} \pi^{2} \mu_{0}^{4} g^{-4} d^{4} z
$$

The integral over the collective coordinates $z^{\mu}$ will yield the total volume of space-time, if no massless fermions are present. If there are massless fermions, then we must include the sources $\mathcal{O}$, which break the translation invariance. In that case the $z$ integration is rather like the integration over the location of an interaction vertex in a Feynman diagram in coordinate configuration, as we will see in Sec. XIV.

\section{COLLECTIVE COORDINATES: 2. DILATATIONS}

From the quantum numbers of the zero eigenstate (8.2) we deduce its angular and index dependence:

$$
A_{\mu}^{a \mathrm{qu}}=\eta_{a \mu \nu} x^{\nu}\left(1+x^{2}\right)^{-2} .
$$

This is a pure infinitesimal dilatation of the classical solution:

$$
A_{\mu}^{a \text { qu }}=\left.\frac{g}{4} \frac{\partial}{\partial \rho}\left(\frac{2}{g} \frac{\eta_{a \mu \nu} x^{\nu}}{x^{2}+\rho^{2}}\right)\right|_{\rho=1} .
$$

Thus, going from the integration variable $A^{\text {qu }}$ in this direction to the collective variable $\rho$, we need a Jacobian factor:

$$
\mathrm{D} A_{\mu}^{a \mathrm{qu}} \rightarrow \frac{4}{g} d \rho\left[\frac{1}{\pi} \int\left(A_{\mu}^{a \mathrm{qu}}\right)^{2} d^{4} x\right]^{1 / 2}
$$

The norm of the solution (10.1) is

$$
\int\left(A_{\mu}^{a \mathrm{qu}}\right)^{2} d^{4} x=\pi^{2} .
$$

Thus, from this mode we obtain the factor

$$
\frac{4 \pi}{g}\left(\frac{\mu_{0}^{2}}{\pi}\right)^{1 / 2} d \rho=4 \pi^{1 / 2} \mu_{0} g^{-1} d \rho
$$

at $\rho=1$. Our system is not scale invariant because of the nontrivial renormalization-group behavior. The complete $\rho$ dependence for $\rho \neq 1$ will be deduced from simple dimensional arguments (including renormalization group) in Sec. XII.

\section{GLOBAL GAUGE ROTATIONS AND THE GAUGE CONDITION}

Discussion of the legitimacy of the background gauge-fixing term has been deliberately postponed to this section, because we wanted to derive first the existence of the three anomalous zero eigenstates (8.3). They have the explicit form 
(arbitrary normalization)

$$
\psi_{\mu}^{a}(b)=2 \eta_{a k \mu} \bar{\eta}_{b k \lambda} x^{\lambda}\left(1+x^{2}\right)^{-2} .
$$

They are a pure gauge artifact

$$
\begin{aligned}
& \psi_{\mu}^{a}(b)=D_{\mu} \psi^{a}(b), \\
& \psi^{a}(b)=\eta_{a k \nu} \bar{\eta}_{b k \lambda} x^{\nu} x^{\lambda}\left(1+x^{2}\right)^{-1},
\end{aligned}
$$

but $\psi^{a}(b)$ is not square-integrable. What is going on? Note that $\psi^{a}(b)$, since they are $x$ dependent, do not generate a closed algebra of gauge rotations. They may not be replaced by a collective coordinate for global isospin rotations. To analyze this situation we first go back to a background-independent gauge-fixing term,

$$
\begin{aligned}
C_{1}^{a}(x) & =\alpha \partial_{\mu}\left(A_{\mu}^{a \mathrm{c} 1}+A_{\mu}^{a \mathrm{qu}}\right) \\
& =\alpha \partial_{\mu} A_{\mu}^{a \mathrm{qu}},
\end{aligned}
$$

where $\alpha$ is a free parameter. In this gauge we know exactly how to handle all zero eigenmodes: There are five for translations and dilatations and also three for global isospin rotations because global isospin is still an invariance in this gauge. To understand the latter we put the system in a large spherical box with volume $V$ and assume that all (vector and ghost) fields vanish on the boundary. Let

$$
\Lambda_{1}^{a}(b, x)=\delta^{a b}
$$

generate an infinitesimal global isospin rotation. Then there is a zero eigenmode:

$$
\begin{aligned}
\psi_{1 \mu}^{a}(b) & =D_{\mu} \Lambda_{1}^{a}(b) \\
& =2 \epsilon_{a c b} \eta_{c \mu \nu} x^{\nu}\left(1+x^{2}\right)^{-1} .
\end{aligned}
$$

The subscript 1 is to remind us that this is a solution in the gauge $C_{1}$. Similarly as in the foregoing two sections, we can replace the integral over $D A_{\mu}^{a}$ by an integral over the collective coordinates $d \Lambda(b)$ by inserting the corresponding Jacobian factor

$$
D A_{\mu}^{a} \rightarrow \int \prod_{b} d \Lambda(b)(\mathscr{N} / \pi)^{1 / 2}
$$

with

$$
\Re=\int\left[\psi_{1 \mu}^{a}(b)\right]^{2} d^{4} x,
$$

which diverges as the volume $V$ of space-time goes to infinity. The integral over the gauge rotation is just the volume of the group and yields

$$
\int \prod_{b} d \Lambda^{b}=\frac{8 \pi^{2}}{g^{3}}
$$

where the factor $g^{-3}$ comes from our normalization of $\Lambda_{1}$ in (11.6). Thus, in this gauge, the zero eigenmode yields the factor

$$
\frac{8 \pi^{2}}{g^{3}}\left(\frac{\mathscr{N}}{\pi}\right)^{3 / 2}
$$

Now we will study different gauges, and for that we need to change the boundary condition (we know from Sec. III that that will not affect the finite eigenvalues, but the zero eigenmodes change) into a gauge-invariant one: The ghosts $\phi$ and gauge generators $\Lambda$ must satisfy

$$
\frac{\partial \phi}{\partial r}(R)=\frac{\partial \Lambda}{\partial r}(R)=0,
$$

where $R$ is the radius of the box, and the vector fields

$$
A_{r}(R)=0, \frac{\partial}{\partial r} A_{\|}(R)=0
$$

where $A_{\|}$is the vector component parallel to the boundary.

Now observe the following. The gauge term $C_{1}$, Eq. (11.4), does not fix the gauge completely which can be seen in two ways: (a) Global isospin rotations are still an invariance; (b) one component of the gauge term $C_{1}$ is identically zero:

$$
\int C_{1}^{a}(x) d^{4} x=0 .
$$

This leaves us the possibility of adding a constant to $C_{1}$, which is orthogonal to it, with which we fix the remaining global gauge

$$
\begin{aligned}
C_{2}^{a}(x)= & \alpha \partial_{\mu} A_{\mu}^{a \mathrm{qu}} \\
& -\kappa \sum_{b} \delta^{a b} \int \psi_{1 \mu}^{c}(b, y) A_{\mu}^{c \text { qu}}(y) d^{4} y
\end{aligned}
$$

with $\alpha, \kappa$ free parameters and $\psi_{1 \mu}^{a}(b, y)=D_{\mu} \delta^{a b}$, as in (11.6). The integral over group space is now replaced by a Gaussian integral. The Gaussian volume is corrected for by the Faddeev-Popov ghost,

$$
\begin{aligned}
\mathcal{L}_{2}^{\mathrm{gh}}=\phi_{a}^{*}(x)\left[\alpha \partial_{\mu} D_{\mu} \phi^{a}(x)\right. \\
\left.-\kappa \sum_{b} \delta^{a b} \int \psi_{1 \mu}^{c}(b, y) D_{\mu} \phi^{c}(y) d^{4} y\right]
\end{aligned}
$$

so that the combined contribution of vector fields and ghosts is now independent of $\alpha$ and $\kappa$ : The zero eigenvalues are replaced by

$$
\lambda_{2}^{\text {vector }}=\kappa^{2} V \Re
$$

and

$$
\lambda_{2}^{\text {ghost }}=\kappa \Re .
$$

(Remember that the ghost, with the new boundary 
condition, has now an eigenstate $\phi=$ constant.) Thus, instead of (11.10), this gauge gives

$$
\left(\lambda_{2}^{\text {ghost }}\right)^{3}\left(\lambda_{2}^{\text {vector }}\right)^{-3 / 2}=(\mathscr{N} / V)^{3 / 2} .
$$

Conclusion: If the redundant eigenmodes are fixed by an additional component in the gauge-fixing term, then a correction factor is needed: Equation (11.10) divided by (11.17):

$$
\frac{8 \pi^{2}}{g^{3}}\left(\frac{V}{\pi}\right)^{3 / 2}
$$

Here $V$ is the volume of the spherical box.

The background gauge $C_{4}=D_{\mu} A_{\mu}^{a \text { qu }}$ has a problem similar to the gauge $C_{1}$. Both the ghost (under the new boundary condition) and the vector field have one eigenvalue that vanishes like $1 / V$ as $V \rightarrow \infty$ (not $1 / R^{2}$, as the other eigenvalues). Let $\psi_{4}^{a}(b)$ be the three ghost eigenstates and $\psi_{4 \mu}^{a}(b)=D_{\mu} \psi_{4}^{a}(b)$ be the vector ones. Let $\lambda_{4}$ be the ghost eigenvalue

$$
D^{2} \psi_{4}^{a}(b)=-\lambda_{4} \psi_{4}^{a}(b) .
$$

It is easy to see that

$$
\lambda_{4}=\frac{\int_{V}\left[\psi_{4 \mu}^{a}(x)\right]^{2} d^{4} x}{\int_{V}\left[\psi_{4}^{a}(x)\right]^{2} d^{4} x} .
$$

From (11.1) and (11.3) we see that this is $O(1 / V)$. It is safer to have a gauge condition that fixes this gauge degree of freedom as $V \rightarrow \infty$,

$$
\begin{aligned}
C_{3}(x)= & \alpha D_{\mu} A_{\mu}^{a \mathrm{qu}}(x) \\
& -\kappa \sum_{b} \psi_{4}^{a}(b, x) \int \psi_{4 \mu}^{c}(b, y) A_{\mu}^{c \mathrm{qu}}(y) d^{4} y,
\end{aligned}
$$

although the result, (11.25), will turn out to remain the same even if $\kappa=0, \alpha=1$. The ghost Lagrangian is

$$
\begin{aligned}
\mathcal{L}_{3}^{\mathrm{gh}}=\phi_{a}^{*}(x)[ & \alpha D^{2} \phi_{a}(x) \\
& \left.-\kappa \sum_{b} \psi_{4}^{a}(b, x) \int \psi_{4 \mu}^{c}(b, y) D_{\mu} \phi^{c}(y) d^{4} y\right] .
\end{aligned}
$$

In this gauge

$$
\lambda_{3}^{\text {vector }}=\lambda_{4}\left(\alpha+\kappa \int\left[\psi_{4}^{a}(x)\right]^{2} d^{4} x\right)^{2}
$$

and

$$
\lambda_{3}^{\text {ghost }}=\lambda_{4}\left(\alpha+\kappa \int\left[\psi_{4}^{a}(x)\right]^{2} d^{4} x\right),
$$

where no summation over $b$ is implied. In this gauge we find the contribution from the lowest eigenmodes:

$$
\left(\lambda_{3}^{\text {ghost }}\right)^{3}\left(\lambda_{3}^{\text {vector }}\right)^{-3 / 2}=\lambda_{4}{ }^{3 / 2} .
$$

Using

$$
\begin{aligned}
& \int\left[\psi^{a}(x)\right]^{2} d^{4} x=V, \\
& \int\left[\psi_{\mu}^{a}(x)\right]^{2} d^{4} x=4 \pi^{2},
\end{aligned}
$$

we find

$$
\lambda_{4}=4 \pi^{2} / V \text {. }
$$

Thus, together with the correction factor (11.18), we find the correct contribution for the three eigenmodes (9.3), together with that of their regulator:

$$
\frac{8 \pi^{2}}{g^{3}}\left(\frac{V \mu_{0}^{2}}{\pi}\right)^{3 / 2}\left(\frac{4 \pi^{2}}{V}\right)^{3 / 2}=2^{6} \pi^{7 / 2} \mu_{0}^{3} g^{-3}
$$

\section{ASSEMBLING THE VECTOR, SCALAR, AND SPINOR TERMS}

The eight zero-eigenvalue modes for the vector field give the factors (9.6), (10.5), and (11.28). Multiplying these gives

$$
2^{14} \pi^{6} g^{-8} \mu_{0}^{8} d^{4} z d \rho
$$

for $\rho=1$ (later we will find the $\rho$ dependence).

The contributions from the nonvanishing eigenmodes both for the scalar and for the vector fields are essentially contained in formula (7.6). As we saw before, the vector fields, combined with the Faddeev- Popov ghost, together count as two real, or one complex, scalar with $t=1$.

Let there be $N^{s}(t)$ scalar multiplets for each isospin $t$, where each complex scalar multiplet counts as one, and each real scalar multiplet counts as one-half. Then from (7.6) we obtain the total contribution from vector and scalar nonzero modes:

$$
\frac{\Pi_{0}}{\Pi}=\exp \left\{-\left[\frac{2}{3}+\frac{1}{6} \sum_{t} N^{s}(t) C(t)\right] \ln \mu_{0}-\alpha(1)-\sum_{t} N^{s}(t) \alpha(t)\right\} .
$$

Here

$$
C(t)=\frac{2}{3} t(t+1)(2 t+1)
$$


and

$$
\alpha(t)=C(t)\left[2 R-\frac{1}{6} \ln 2+\frac{1}{2} \sum_{s=1}^{2 t+1} s(2 t+1-s)\left(s-t-\frac{1}{2}\right) \ln s-\frac{1}{6} t(t+1)-\frac{1}{9}\right] .
$$

The numerical values for $C(t)$ and $\alpha(t)$ are listed in Table I. Combining (12.1), (12.2), and the classical action (2.8) gives the total amplitude in the absence of fermions:

$$
2^{14} \pi^{6} g^{-8} \frac{d^{4} z d \rho}{\rho^{5}} \exp \left\{-\frac{8 \pi^{2}}{g^{2}\left(\mu_{0}\right)}+\ln \left(\mu_{0} \rho\right)\left[\frac{22}{3}-\frac{1}{6} \sum_{t} N^{s}(t) C(t)\right]-\alpha(1)-\sum_{t} N^{s}(t) \alpha(t)\right\} .
$$

Note that the coefficient multiplying $\ln \mu_{0}$ coincides with the usual Callan-Symanzik $\beta$ coefficient for $g^{2}\left(\mu_{0}\right)$ in such a way that $(12.5)$ becomes independent of the subtraction point $\mu_{0}$ if we choose $g^{2}\left(\mu_{0}\right)$ to obey the Gell-Mann-Low equation. We now also insert the $\rho$ dependence if $\rho \neq 1$ by straightforward dimensional analysis.

The interpretation of (12.5) is best given in the language of path integrals: If $|0\rangle$ is the vacuum, and $|\tilde{0}\rangle$ is the gauge-rotated vacuum, then (12.5) is the total contribution to $\langle\tilde{0} \mid 0\rangle$ from all paths in Euclidean space that have a pseudoparticle located at $z$ within $d^{4} z$, having a scale between $\rho$ and $\rho+d \rho$. The fermions can be introduced in two ways:

(i) If they have a mass $m \ll 1 / \rho$ then only the lowest eigenvalue will depend critically on $m$.

(ii) If they are rigorously massless then the lowest eigenvalue depends critically on the external source $d$.

In this paper we limit ourselves only to fermions with isospin $t=\frac{1}{2}$. In case (i) the contribution of the lowest modes will simply be

$$
\left(\frac{m}{\mu_{0}}\right)^{N^{f}}
$$

The nonvanishing eigenmodes are again obtained from (7.6), which represents the eigenvalues of $\mathfrak{M}_{\psi}^{2}$. Now we wish to compute det $\mathfrak{T}_{\psi}$ and we take into account that the Dirac field has four components. Thus the fermion nonvanishing eigenmodes will give

$$
\exp \left[\frac{N^{f}}{3} C\left(\frac{1}{2}\right) \ln \mu_{0}+2 N^{f} \alpha\left(\frac{1}{2}\right)\right] .
$$

Together with $(12.6)$ we find the total fermion factor that multiplies (12.5),

$$
\rho^{N^{f}} m^{N^{f}} \exp \left[-\frac{2}{3} N^{f} \ln \left(\mu_{0} \rho\right)+2 N^{f} \alpha\left(\frac{1}{2}\right)\right],
$$

where we again inserted the factors $\rho$ as they follow from dimensional arguments. Note that the well-known Callan-Symanzik $\beta$ coefficient for $g^{2}\left(\mu_{0}\right)$ again matches the term in front of $\ln \mu_{0}$. Equations (12.5) and (12.8) could be used as a starting point for a strong-interaction color theory where Euclidean pseudoparticles form a plasmalike statistical ensemble. ${ }^{7}$ For that, one also needs to extend from $\mathrm{SU}(2)$ to $\mathrm{SU}(3)$. We will not do that in this paper.

In Sec. XIV we consider case (ii). In that case $m$ must be replaced by the eigenvalue of the lowest mode as it is perturbed by the source insertion.

\section{DIMENSIONAL RENORMALIZATION}

The regulators used in Secs. VII-XII are what we call fixed mass Pauli-Villars regulators and they only make sense in the background-field formalism. They are given by the Lagrangians (4.7)-(4.9). In this section we wish to switch to another regulator scheme which is much more widely used in gauge theories: the dimensional method. ${ }^{8}$ Let us emphasize again that if one switches to another regulator, then that affects the definition of $g(\mu)$ and that influences our calculation by an overall constant. We know that in the dimensional procedure the limit of large cutoff is replaced by a limit $n \rightarrow 4$, where $n$ is the number of space-time dimensions, roughly in the following way:

$$
\ln \Lambda \rightarrow \frac{1}{4-n}+\text { finite. }
$$

In (12.5) and (12.7) the regulator mass $\mu_{0}$ plays the role of the cutoff $\Lambda$. Clearly then, the finite part in (13.1) will be relevant. In this section we derive that finite part, in ordinary perturbation

TABLE I. Numerical values of the coefficients $C(t)$ and $\alpha(t)$ as they occur in the text.

\begin{tabular}{cclr}
\hline \hline$t$ & $C(t)$ & \multicolumn{2}{c}{$\alpha(t)$} \\
\hline 0 & 0 & 0 & $=0.145873$ \\
$\frac{1}{2}$ & 1 & $2 R-\frac{1}{6} \ln 2-\frac{17}{72}$ & $=0.443307$ \\
1 & 4 & $8 R+\frac{1}{3} \ln 2-\frac{16}{9}$ & \\
$\frac{3}{2}$ & 10 & $20 R+4 \ln 3-\frac{5}{3} \ln 2-\frac{265}{36}=0.853182$ \\
$R=\frac{1}{12}(\ln 2 \pi+\gamma)+\frac{1}{2 \pi^{2}} \sum_{2}^{\infty} \frac{\ln s}{s^{2}}=0.248754477033784$ \\
\hline \hline
\end{tabular}


theory. It corresponds to a finite counterterm in the Lagrangian. It is easy to compute this finite counterterm when, again, one makes use of the background fields. There are some diagrams to be computed and the rest is algebra. This algebra is identical to the algebra devised in Ref. 9. Symmetry arguments restrict the possible form of the finite counterterm in just two independent terms, $X^{2}$ and $Y_{\mu \nu} Y_{\mu \nu}$, in the language of Ref. 9. The first of these is obtained by comparing the integral

$$
\frac{1}{(2 \pi)^{n}} \int d^{n} k \frac{1}{\left(k^{2}+\mu_{0}^{2}\right)^{2}}
$$

in the limits $\mu_{0} \rightarrow \infty$ and $n \rightarrow 4$. For definiteness, we specify the theory at $n \neq 4$ dimensions: All trivial factors $(2 \pi)^{4}$ must also be replaced by $(2 \pi)^{n}$, which leads to the factor $(2 \pi)^{-n}$ in (13.2).

The integral (13.2) is in this limit

$\frac{1}{(4 \pi)^{2}}\left[\frac{2}{4-n}-\gamma-2 \ln \mu_{0}+\ln 4 \pi+O(4-n)+O\left(\frac{1}{\mu_{0}^{2}}\right)\right]$

where $\gamma$ is again Euler's constant. So here

$$
\ln \mu_{0} \rightarrow \frac{1}{4-n}-\frac{1}{2} \gamma+\frac{1}{2} \ln 4 \pi \text {. }
$$

The coefficient in front of $Y_{\mu \nu} Y_{\mu \nu}$ is obtained in the same way by comparing the integral

$$
\frac{1}{(2 \pi)^{n}} \int d^{n} k \frac{\left(k^{2}\right)^{2}}{\left(k^{2}+\mu_{0}^{2}\right)^{4}}
$$

in the two limits. This time we get

$$
\begin{aligned}
\frac{1}{(4 \pi)^{2}}\left[\frac{2}{4-n}-\gamma-2 \ln \mu_{0}+\ln 4 \pi-\frac{5}{6}+O(4\right. & -n) \\
& \left.+O\left(\frac{1}{\mu_{0}^{2}}\right)\right] .
\end{aligned}
$$

Thus

$$
\ln \mu_{0} \rightarrow \frac{1}{4-n}-\frac{1}{2} \gamma+\frac{1}{2} \ln 4 \pi-\frac{5}{12}
$$

So we get different answers in the two different cases and we must find out which of the coefficients $\ln \mu_{0}$ come from which of the terms, either the $X^{2}$ or the $Y_{\mu \nu} Y_{\mu \nu}$ term. That turns out to be easy. The $Y_{\mu \nu} Y_{\mu \nu}$ terms only come from the covariant derivatives in (4.7)-(4.9), whereas all other interactions, including the one proportional to $\sigma_{\mu \nu} G_{\mu \nu}$ in (4.8), occur in the $X^{2}$ terms. Thus, the substitution (13.7) must be made for the scalar fields and for those contributions of the vector and spinor fields that come from their kinetic terms. Those are exactly the contributions that make up the $\ln \mu_{0}$ in (7.6). The substitution (13.4) must be made for the remaining $\ln \mu_{0}$ terms, which are exactly all $\ln \mu_{0}$ contributions that came from the zero eigenmodes (12.1).

In conclusion, (12.5) is to be replaced by

$$
2^{14} \pi^{6} g^{-8} \rho^{-5} d^{4} z d \rho \exp \left\{-\frac{8 \pi^{2}}{g_{B}^{2}(n)}+\left(\ln \rho+\frac{1}{4-n}\right)\left[\frac{22}{3}-\frac{1}{6} \sum_{t} N^{s}(t) C(t)\right]+A-\sum_{t} N^{s}(t) A(t)\right\},
$$

with

$$
A=-\alpha(1)+\frac{11}{3}(\ln 4 \pi-\gamma)+\frac{5}{18}=6.998435
$$

and

$$
A(t)=\alpha(t)+\frac{1}{12}\left(\ln 4 \pi-\gamma-\frac{5}{6}\right) C(t) .
$$

Numerically,

$$
\begin{aligned}
& A(0)=0, \quad A\left(\frac{1}{2}\right)=0.239246, \quad A(1)=0.816799, \\
& A\left(\frac{3}{2}\right)=1.786912 .
\end{aligned}
$$

Similarly for the fermion factor

$$
\rho^{N^{f}} m^{N^{f}} \exp \left[-\frac{2}{3} N^{f}\left(\ln \rho+\frac{1}{4-n}\right)-N^{f} B\right]
$$

with

$$
\begin{aligned}
B & =-2 \alpha\left(\frac{1}{2}\right)+\frac{1}{3}\left(\ln 4 \pi-\gamma+\frac{5}{12}\right) \\
& =0.498412 .
\end{aligned}
$$

If we define the subtracted coupling constant as in Ref. 10,

$$
g_{B}(n)=\mu^{4-n}\left(g_{R}^{D}(\mu)+\frac{a_{1}}{n-4}+\cdots\right)
$$

with $a_{1}$ depending only on $g_{R}$ but not on $n$ or $\mu$, then we can make the following replacements in (13.8) and (13.11):

$$
\begin{aligned}
& g_{B}(n) \rightarrow g_{R}^{D}(\mu), \\
& \ln \rho+\frac{1}{4-n} \rightarrow \ln (\rho \mu) .
\end{aligned}
$$

Here the superscript $D$ stands for the dimensional procedure which defines $g_{R}^{D}$. We see that the expression in terms of $g_{R}^{D}(\mu)$ differs slightly from the one in terms of $g\left(\mu_{0}\right)$. 


\section{THE FERMION SOURCE AND THE GREEN'S FUNCTION}

We now consider the fermion zero eigenmode (9.4), and assume that the fermion mass (12.6) vanishes. In that case the source $\partial_{s t}$ in (2.1) must be taken into account, since the lowest eigenvalue will now mainly be determined by this source. We determine the lowest eigenvalues $E(i), i=1, \ldots, N^{f}$ of the operator

$$
\mathfrak{T}_{\psi}=-\gamma_{\mu} D_{\mu} \delta_{s t}+\partial_{s t}
$$

by perturbation theory, taking $\mathcal{J}$ as the small perturbation. The method is the standard one (the author thanks S. Coleman for an enlightening discussion on this point). The unperturbed, degenerate eigenmodes are (taking for simplicity $\rho=1)$

$$
\psi_{s}^{\alpha}(t)=C\left(1+r^{2}\right)^{-3 / 2} u^{\alpha} \delta_{s t},
$$
$\alpha=1,2, s, t=1, \ldots, N^{f}$.

The coefficients $u^{\alpha}$ contain besides the isospin index $\alpha$ a Dirac index. They satisfy

$$
\begin{aligned}
& J_{1}^{a} u^{\alpha}=S_{1}^{a} u^{\alpha}+T_{\alpha \beta}^{a} u^{\beta}=0, \\
& \left(\eta_{a \mu \nu} \gamma_{\mu} \gamma_{\nu}+4 i \tau_{a}\right) u=0
\end{aligned}
$$

or

and

$$
\gamma_{5} u=-u \text {. }
$$

The coefficient $C$ is determined by normalizing $\psi$,

$$
\begin{aligned}
C^{-2} & =u^{*} u \int d^{4} x\left(1+x^{2}\right)^{-3} \\
& =\pi^{2} / 2 \text { if } u^{*} u=1 .
\end{aligned}
$$

Let

$$
\begin{aligned}
H_{s t} & =\left\langle\psi(s)\left|\mathfrak{N}_{\psi}\right| \psi(t)\right\rangle \\
& =\frac{2}{\pi^{2}} \int d^{4} x\left(1+x^{2}\right)^{-3} u_{\alpha}^{*} \mathcal{J}_{s t}(x) u^{\alpha},
\end{aligned}
$$

then $E(i)$ are the $N^{f}$ eigenvalues of $H$. We wish to compute

$$
\begin{aligned}
\prod_{i} E(i) & =\operatorname{det} H \\
& =\left(\frac{2}{\pi^{2}}\right)^{N^{f}} \operatorname{det}_{s t} \int d^{4} x\left(1+x^{2}\right)^{-3} u_{\alpha}^{*} \partial_{s t}(x) u^{\alpha} .
\end{aligned}
$$

For large $x^{2}$, this amplitude has exactly the space-time structure of an $N^{f}$-point Green's function, where each source point is connected to the origin by two fermion lines. The integral over the collective coordinate $z$ [which is at the origin in Eq. (14.7)] will correspond to the integration in coordinate configuration over the vertex variable.

Equation (14.7) should really be considered as our final result for the space-time dependence of the fermion Green's function. But it would be enlightening if we could represent it in terms of an effective Lagrangian.

We found that the effective Lagrangian can best first be written in the form

$$
\mathcal{L}^{\mathrm{eff}}=C \prod_{s=1}^{N f}\left(\bar{\psi}_{s} \omega\right)\left(\bar{\omega} \psi_{s}\right),
$$

where $\omega$ is some fixed Dirac spinor with isospin $\frac{1}{2}$. Owing to Fermi statistics, the various terms of the determinant in (14.7) will arise with the appropriate minus signs so that we may limit ourselves to sources $\mathcal{J}_{s t}$ that are diagonal in $s$ and $t$ :

$$
\delta_{s t}=\mathcal{J}(x) \delta_{s t} .
$$

Here $\mathcal{d}$ may still contain Dirac matrices.

In the presence of this source, the amplitude from the effective interaction (14.8) would be

$$
C \prod_{s}\left[-\bar{\omega} S_{F}(x) \mathcal{J}(x) S_{F}(-x) \omega\right],
$$

where the minus sign comes from the Fermi statistics and the $S_{F}(x)$ are the Dirac propagators for massless fermions in coordinate configuration,

$$
S_{F}(x)=\frac{\gamma x}{2 \pi^{2}\left(x^{2}\right)^{2}} .
$$

Comparing this with (14.7), at large $x^{2}$, we find that we must require (leaving aside temporarily the other contributions to the overall constant)

$$
\begin{aligned}
& \left(\gamma x \omega_{\alpha}\right)\left(\bar{\omega}_{\alpha} \gamma x\right)=x^{2} u_{\alpha} \bar{u}_{\alpha}, \\
& C=\left(8 \pi^{2}\right)^{N f},
\end{aligned}
$$

where we may sum over the isospin index $\alpha$ but not over the Dirac components. Now from (14.3) one can derive

$$
\sum_{\alpha} u_{\alpha} \bar{u}_{\alpha}=\frac{1}{2}\left(1-\gamma_{5}\right),
$$

so we must require the $\omega_{\alpha}$ to be such that

$$
\sum_{\alpha} \omega_{\alpha} \bar{\omega}_{\alpha}=\frac{1}{2}\left(1+\gamma_{5}\right) \text {. }
$$

Thus, the $\omega_{\alpha}$ are some parity reflection of $u_{\alpha}$.

There is clearly no gauge-invariant solution to (14.14), so our effective Lagrangian (14.8) is apparently not gauge invariant. But note that we only wish to reproduce the amplitude (14.7) for gauge-invariant currents $\mathcal{d}_{s t}$. Thus, any gauge rotation of (14.8) does the same job. We get a gauge-invariant $\mathcal{L}^{\text {eff }}$ if we average over the whole 
group of gauge rotations:

$$
\mathcal{L}^{\mathrm{eff}}=C\left\langle\prod_{s=1}^{N^{f}}\left(\bar{\psi}_{s} \omega\right)\left(\bar{\omega} \psi_{s}\right)\right\rangle,
$$

where the brackets \langle\rangle denote the average for all gauge rotations of $\omega$. We can then derive

$$
\left\langle\omega_{\alpha} \bar{\omega}_{\beta}\right\rangle=\frac{1}{4} \delta_{\alpha \beta}\left(1+\gamma_{5}\right),
$$

and, for instance,

$$
\begin{aligned}
\left\langle\prod_{s=1}^{2}\left(\bar{\psi}_{s} \omega\right)\left(\bar{\omega} \psi_{s}\right)\right\rangle= & \frac{1}{24}\left(2 \delta_{\alpha_{1}}^{\beta_{1}} \delta_{\alpha_{2}}^{\beta_{2}}-\delta_{\alpha_{1}}^{\beta_{2}} \delta_{\alpha_{2}}^{\beta_{1}}\right) \epsilon^{s t} \\
& \times \bar{\psi}_{1}^{\alpha_{1}}\left(1+\gamma_{5}\right) \psi_{s}^{\beta_{1}} \bar{\psi}_{2}^{\alpha_{2}}\left(1+\gamma_{5}\right) \psi_{t}^{\beta_{2}} .
\end{aligned}
$$

The Lagrangian (14.15) only acts on the lefthanded spinors. The parity-reflected Euclidean pseudoparticles will give a similar contribution acting on the right-handed spinors only. So in total we get $\mathscr{L}^{\mathrm{eff}}$ of $(14.15)$ plus its Hermitian conjugate.

Note that we obtain products of fermion fields, such as (14.17), that violate only chiral $U(1)$ invariance. They have the chiral-symmetry properties of the determinant of an $N^{f} \times N^{f}$ matrix in flavor space and are therefore still invariant under chiral SU $\left(N^{f}\right) \times \mathrm{SU}\left(N^{f}\right)$. The symmetry violation is associated with an arbitrary phase factor $e^{ \pm i \omega}$ in front of the effective Lagrangians. If other mass terms or interaction terms occur in the Lagrangian that also violate chiral $\mathrm{U}(1)$, then they may have a phase factor different from these. We then find that our effective Lagrangian may violate $P$ invariance, whereas $C$ invariance is maintained. Thus we find that not only $\mathrm{U}(1)$ invariance but also $P C$ invariance can be violated by our effect.

\section{CONVERGENCE OF THE $\rho$ INTEGRATION}

The entire expression that we now have for the effective Lagrangian is

$$
\begin{aligned}
\mathscr{L}^{\mathrm{eff}}(z) d^{4} z=2^{14+3 N^{f}} \pi^{6+2 N^{f}} g^{-8} d^{4} z \int \rho^{-5+3 N^{f}} d \rho \exp \{ & -\frac{8 \pi^{2}}{\left[g_{R}^{D}(\mu)\right]^{2}}+\ln (\mu \rho)\left[\frac{22}{3}-\frac{1}{6} \sum_{t} N^{s}(t) C(t)-\frac{2}{3} N^{f}\right] \\
& \left.+A-\sum_{t} N^{s}(t) A(t)-N^{f} B\right\}\left\langle\prod_{s=1}^{N^{f}}\left(\bar{\psi}_{s} \omega\right)\left(\bar{\omega} \psi_{s}\right)\right\rangle+\text { H.c. }
\end{aligned}
$$

with

$$
\left\langle\omega_{\alpha} \bar{\omega}_{\beta}\right\rangle=\frac{1}{4} \delta_{\alpha \beta}\left(1+\gamma_{5}\right) \text {, etc. , }
$$

and the numbers $A, A(t), B, C(t)$ as defined before.

The $\rho$ dependence has been changed because the effective Lagrangian (14.8) is not dimensionless.

We see that this integral converges as $\rho \rightarrow 0$ (except when there are very many scalars). But there is an infrared divergence as $\rho \rightarrow \infty$. In an unbroken color gauge theory for strong interactions this is just one of the various infrared disasters of the theory to which we have no answer. But in a weak-interaction theory it is expected that the Higgs field provides for the cutoff. Let there be a Higgs field with isospin $q$ and vacuum expectation value $F$. Let its contribution to the original Lagrangian be

$$
\mathcal{L}^{H}=-D_{\mu} \Phi^{*} D_{\mu} \Phi-V(\Phi) .
$$

Formally, no classical solution exists now, because the Higgs Lagrangian tends to add to the total action of the pseudoparticle a contribution proportional to $F^{2}$, but this can always be re- duced by scaling to smaller distances, until the action reaches the usual value $8 \pi^{2} / g^{2}$ when the field configuration is singular.

On the other hand, it is clear that the quantum corrections, as can be seen in (15.1), act in the opposite way. There must be a region of values for $\rho$ where the quantum effects compete with the effects due to the Higgs fields.

To handle this situation rigorously we alter slightly the philosophy of Sec. II. In Euclidean space it is not compulsory to consider only those classical fields for which the action is stationary. We will now look at approximate solutions of the classical equations, so that the total action is only a slowly varying function of one collective parameter, $\rho$.

We simply postulate the gauge field $A$ to have the same configuration as before, with certain value for $\rho$, and now choose the Higgs field configuration in such a way that the total action is extreme. Only those inf initesimal variations that are pure scale transformations do not leave the action totally invariant, but nevertheless the parameter $\rho$ gets the full 
treatment as a collective variable.

As will be verified explicitly, the dominant values for $\rho$ will be those where the quantum effects and the Higgs contribution are equally important. Since the quantum effects are small we expect that there

$$
\rho \ll 1 / M_{H},
$$

which implies that the Higgs particle may be considered as approximately massless. Let us scale toward

$$
\rho=1, \quad|F| \sim 1, \quad M_{H}^{2} \sim \lambda F^{2} \ll 1 .
$$

The equation for this field will be approximately

$$
\begin{aligned}
& D^{2} \Phi=0, \\
& \Phi^{2}(r \rightarrow \infty)=F^{2} .
\end{aligned}
$$

The solution to that is a zero-eigenvalue mode of the familiar operator (2.22):

$$
\begin{aligned}
& j_{1}=0, l=j_{2}=q, \\
& |\Phi|=\left(\frac{x^{2}}{1+x^{2}}\right)^{q}|F| .
\end{aligned}
$$

The contribution to the classical action is

$$
S^{H}=\int\left[-D_{\mu} \Phi^{*} D_{\mu} \Phi-V(\Phi)\right] d^{4} x .
$$

The first term is (observing that $x_{\mu} A_{\mu}^{\mathrm{c} 1}=0$ )

$$
\begin{aligned}
-\int_{V} \partial_{\mu}\left(\Phi^{*} D_{\mu} \Phi\right) d^{4} x & =-\int_{s} \Phi^{*} \partial_{r} \Phi d^{3} x \\
& =-4 \pi^{2} q F^{2} .
\end{aligned}
$$

The second term in (15.6) is of order $\lambda F^{4}$, where $\lambda$ is a small coupling constant. If we scale back to arbitrary $\rho$, then the Higgs field factor in the total expression is

$$
\exp S^{H}=\exp \left[-4 \pi^{2} q F^{2} \rho^{2}-O\left(\lambda F^{4} \rho^{4}\right)\right] .
$$

We see that the second term in the exponent may be neglected at first approximation.

Thus (15.8) multiplies the integrand in (15.1) and the $\rho$ integration is now completely convergent. The integration over $\rho$ yields a factor

$$
\frac{1}{2}\left(4 \pi^{2} q F^{2}\right)^{2-(3 / 2) N_{-C} C} \Gamma\left(\frac{3}{2} N^{f}+C-2\right),
$$

where

$$
C=\frac{11}{3}-\frac{1}{12} \sum_{t} N^{s}(t) C(t)-\frac{1}{3} N^{f}
$$

\section{ACKNOWLEDGMENT}

The author wishes to thank S. Coleman, R. Jackiw, C. Rebbi, and all other theorists at
Harvard for their hospitality, encouragement, and discussions during the completion of this work.

\section{APPENDIX: PROPERTIES OF THE $\eta$ SYMBOLS}

The group $\mathrm{SO}(4)$ is locally equivalent to $\mathrm{SO}(3)$ $\times \mathrm{SO}(3)$. The antisymmetric tensors $A_{\mu \nu}$ in $\mathrm{SO}(4)$ having six components form a $3+3$ representation of $\mathrm{SO}(3) \times \mathrm{SO}(3)$. The self-dual tensors

$$
A_{\mu \nu}=\frac{1}{2} \epsilon_{\mu \nu \alpha \beta} A_{\alpha \beta}
$$

transform as 3 -vectors of one $\mathrm{SO}(3)$ group. We now define the $\eta$ symbols, in a way very similar to the Dirac $\gamma$ matrices:

$$
\begin{aligned}
& A_{\mu \nu}=\eta_{a \mu \nu} A_{a}, \\
& a=1,2,3, \quad \mu, \nu=1, \ldots, 4
\end{aligned}
$$

is a covariant mapping of $\mathrm{SO}(3)$ vectors on selfdual $\mathrm{SO}(4)$ tensors. A convenient representation is

$$
\begin{aligned}
& \eta_{a \mu \nu}=\epsilon_{a \mu \nu}, \text { if } \mu, \nu=1,2,3 \\
& \eta_{a 4 \nu}=-\delta_{a \nu}, \\
& \eta_{a \mu 4}=\delta_{a \mu}, \\
& \eta_{a 44}=0 .
\end{aligned}
$$

Let us also define

$$
\bar{\eta}_{a \mu \nu}=(-1)^{6 \mu 4^{+6} \nu 4} \eta_{a \mu \nu} \text {. }
$$

The symbols $\bar{\eta}_{a \mu \nu}$ will then do the same with vectors of the other $\mathrm{SO}(3)$ group and tensors $B_{\mu \nu}$ that are minus their own dual.

We have the following identities:

$$
\begin{aligned}
& \eta_{a \mu \nu}=\frac{1}{2} \epsilon_{\mu \nu \alpha \beta} \eta_{a \alpha \beta}, \quad \bar{\eta}_{a \mu \nu}=-\frac{1}{2} \epsilon_{\mu \nu \alpha \beta} \bar{\eta}_{a \alpha \beta}, \\
& \eta_{a \mu \nu}=-\eta_{a \nu \mu}, \\
& \eta_{a \mu \nu} \eta_{b \mu \nu}=4 \delta_{a b}, \\
& \eta_{a \mu \nu} \eta_{a \mu \lambda}=3 \delta_{\nu \lambda}, \\
& \eta_{a \mu \nu} \eta_{a \mu \nu}=12, \\
& \eta_{a \mu \nu} \eta_{a k \lambda}=\delta_{\mu k} \delta_{\nu \lambda}-\delta_{\mu \lambda} \delta_{\nu k}+\epsilon_{\mu \nu k \lambda}, \\
& \delta_{k \lambda} \eta_{a \mu \nu}+\delta_{k \nu} \eta_{a \lambda \mu}+\delta_{k \mu} \eta_{a \nu \lambda}+\eta_{a \sigma k} \epsilon_{\lambda \mu \nu \sigma}=0, \\
& \eta_{a \mu \nu} \eta_{b \mu \lambda}=\delta_{a b} \delta_{\nu \lambda}+\epsilon_{a b c} \eta_{c \nu \lambda}, \\
& \epsilon_{a b c} \eta_{b \mu \nu} \eta_{c k \lambda}=\delta_{\mu k} \eta_{a \nu \lambda}-\delta_{\mu \lambda} \eta_{a \nu k}-\delta_{\nu k} \eta_{a \mu \lambda}+\delta_{\nu \lambda} \eta_{a \mu k},
\end{aligned}
$$

$$
\begin{aligned}
& \eta_{a \mu \nu} \bar{\eta}_{b \mu \nu}=0 \\
& \eta_{a k \mu} \bar{\eta}_{b k \lambda}=\eta_{a k \lambda} \bar{\eta}_{b k \mu} .
\end{aligned}
$$


*Work supported in part by the National Science Foundation under Grant No. MPS 75-20427.

$\dagger$ On leave from the University of Utrecht.

${ }^{1}$ A. A. Belavin et al., Phys. Lett. 59B, 85 (1975).

${ }^{2}$ G. 't Hooft, Phys. Rev. Lett. 37, 8(1976); R. Jackiw and C. Rebbi, ibid. 37, 172 (1976); Phys. Rev. D 14, 517 (1976); C. Callan, R. Dashen, and D. Gross, Phys. Lett. 63B, 334 (1976). See also F. R。Ore, Jr., Phys. Rev. $\mathrm{D}$ (to be published).

${ }^{3} \mathrm{R}$. Jackiw and S. Coleman (private communication).

${ }^{4}$ J. S. Bell and R. Jackiw, Nuovo Cimento 51, 47 (1969); S. L. Adler, Phys. Rev. 177, 2426 (1969).

${ }^{5} \mathrm{~J}$. L. Gervais and B. Sakita, Phys. Rev. D 11, 2943 (1975); E. Tomboulis, ibid. 12, 1678 (1975).
${ }^{6} \mathrm{~J}$. Honerkamp, Nucl. Phys. B48, 269 (1972); J. Honerkamp, in Proceedings of the Colloquium on Renormalization of Yang-Mills Fields and Applications to Particle Physics, 1972, edited by C. P. Korthals-Altes (C.N.R.S., Marseille, France, 1972).

${ }^{7}$ A. M. Polyakov, Phys. Lett. 59B, 82 (1975).

${ }^{8}$ G. 't Hooft and M. Veltman, Nucl. Phys. B44, 189 (1972); C. G. Bollini and J. J. Giambiagi, Phys. Lett. 40B, 566 (1972); J. F. Ashmore, Lett. Nuovo Cimento 4, 289 (1972); G. 't Hooft and M. Veltman, Report No. CERN 73-9, 1973 (unpublished).

${ }^{9}$ G. 't Hooft, Nucl. Phys. B62, 444. (1973).

${ }^{10} \mathrm{G}$. 't Hooft, Nucl. Phys. B61, 455 (1973). 